\title{
Unprecedented $\mathrm{SnCl}_{2}$ Mediated Cyclization of nitro arenes via $N-N$ bond formation
}

\author{
Devesh Sawant, ${ }^{\mathrm{a}}$ Rishi Kumar, ${ }^{\mathrm{b}}$ Prakas R. Maulik ${ }^{\mathrm{b}}$ and Bijoy Kundu ${ }^{\mathrm{a} *}$ \\ ${ }^{a}$ Medicinal Chemistry Division, Central Drug Research Institute, Lucknow 226 001, India. \\ ${ }^{b}$ Molecular Structural Biology Division, Central Drug Research Institute, Lucknow 226 001, India.
}

Corresponding author e-mail : bijoy_kundu@yahoo.com; Tel. +91-522-2612411-18;

Fax: +91-522-2623405

\section{Table of Contents $\quad$ Page}

1 General experimental $\quad$ S2

2 Experimental Procedure and Spectroscopic Data for 1-(2-Nitrophenyl)- S2 3,4-dihydroisoquinoline [3] and derivatives [3 (b-d)]

2.1 Preparation of phenethylamine $\quad$ S2

2.2 Preparation of N-[2-(3,4-dimethoxyphenyl)ethyl]-2-nitrobenzamide $\quad$ S3

2.3 Preparation of 6,7-dimethoxy-1-(2-nitrophenyl)-3,4-dihydroisoquinoline [1a] S4

2.4 Catalytic reduction of 1a to 2-(6,7-Dimethoxy-3,4-dihydroisoquinolin-1-yl)- S5 phenylamine [2a]

2.5 Basic $\mathrm{SnCl}_{2} \cdot 2 \mathrm{H}_{2} \mathrm{O}$ reduction of 1a to N-[2-(6,7-Dimethoxy-3,4-dihydroisoquinolin- S5 1-yl)-phenyl]hydroxylamine [11a]

2.6 Cyclization of 11a to $3 \mathrm{a}$ in presence of $\mathrm{TsCl} \quad \mathrm{S} 6$

2.7 Intramolecula cyclization of $1 \mathrm{a}$ to 2,3-dimethoxy-5,6-dihydroindazolo[3,2- S6 a]isoquinoline [3a]

$\begin{array}{lll}2.8 & \text { Spectroscopic data of } 3[\mathrm{~b}-\mathrm{d}] & \text { S7 }\end{array}$

3 Experimental Procedure and Spectroscopic Data for 10-methyl-5,12- S7 dihydro-6H-6a,7,12-triaza-indeno[1,2-a]fluorene [14c] and derivatives [14a, 14b, 14d].

3.1 Preparation of N-[2-(1H-Indol-3-yl)-ethyl]-5-methyl-2-nitro-benzamide [12c] S7

3.2 Preparation of 1-(5-Methyl-2-nitro-phenyl)-4,9-dihydro-3H-b-carboline [13c]. S8

3.3 Intramolecular cyclization of $13 \mathrm{c}$ to 10 -methyl-5,12-dihydro-6H-6a,7,12-triaza- S9 indeno[1,2-a]fluorene [14c]

3.4 Spectroscopic data of [14a, 14b, 14d] $\quad$ S10

4 Spectra $\quad$ S11

5 X-ray crystallographic data for compounds 3a and 14a S22

6 References $\quad$ S23 


\section{General Experimental}

Acetonitrile, $N, N$-dimethylformamide (DMF), toluene and methanol were were purchased anhydrous and stored over activated 3 or $4 \AA$ molecular sieves. Triethylamine $\left(\mathrm{Et}_{3} \mathrm{~N}\right)$ was distilled from $\mathrm{CaH}_{2}$ under an atmosphere of argon. Phosphorous oxychloride $\left(\mathrm{POCl}_{3}\right)$ was freshly distilled prior to use. 1-Hydroxybenztriazole (HOBt), dicyclohexylcarbodiimide (DCC), Pd-C, tryptamine and $o$-nitrobenzoic acids were purchased from Aldrich and used directly. Analytical TLC was performed using $2.5 \times 5 \mathrm{~cm}$ plated coated with a $0.25 \mathrm{~mm}$ thickness of silica gel 60F-254 Merck and visualization was accomplished with UV light and iodine. Column chromatography was performed using silica gel 60 Thomas Baker (100-200 mesh). ${ }^{1} \mathrm{H}$ NMR spectra were obtained from 300 or $200 \mathrm{MHz}$ spectrometers and are reported in ppm using tetramethylsilane (TMS, 0.00 $\mathrm{ppm})$ or solvent $\left(\mathrm{CDCl}_{3}=7.26 \mathrm{ppm} ; \mathrm{DMSO}-d_{6}=2.50 \mathrm{ppm}\right)$ as an internal standard. Data are reported as: $[\delta \operatorname{shift}]([\mathrm{br}=$ broad, $\mathrm{s}=$ singlet, $\mathrm{d}=$ doublet, $\mathrm{dd}=$ doublet of doublet, $\mathrm{t}=$ triplet, $\mathrm{q}=$ quartet, $\mathrm{m}=$ multiplet, $\mathrm{o}=$ overlapped $]$, $[J=$ coupling constant in $\mathrm{Hz}]$ and [integration]). Proton-decoupled ${ }^{13} \mathrm{C}$ NMR spectra were recorded at 75 or $50 \mathrm{MHz}$ and are reported in ppm using solvent $\left(\mathrm{CDCl}_{3}=\right.$ $\left.77.0 \mathrm{ppm}, \mathrm{DMSO}-d_{6}=39.43 \mathrm{ppm}\right)$ as an internal standard. Infrared spectra were recorded using an FT-IR instrument with $\mathrm{KBr}$ pellets or neat on $\mathrm{NaCl}$ plates. Analytical HPLC were performed on C-18 $\mu$-bondapak column $(250 \mathrm{~mm}$ x $4.6 \mathrm{~mm}, 10 \mu \mathrm{m})$.with a linear gradient $10-100 \% \mathrm{CH}_{3} \mathrm{CN}$ in water over $35 \mathrm{~min}$, flow rate $1.0 \mathrm{ml} / \mathrm{min}$ and UV detection $220 / 254 \mathrm{~nm}$. Mass spectra were recorded on a Merck MS-8000 spectrometer. Melting points were recorded on Electrothermal digital melting point apparatus and are uncorrected. Hydrogenation was performed on the Parr assembly at $35 \mathrm{psi}$ at room temperature. For characterization purposes hexane was distilled to remove "grease" impurities when used for chromatography. Chloroform $\left(\mathrm{CHCl}_{3}\right)$ used for chromatography contained $0.5-1.0 \% \mathrm{EtOH}$ as stabilizer. Water was triply distilled before using for work up.

\section{Experimental Procedure and Spectroscopic Data for 1-(2- Nitrophenyl)-3,4-dihydroisoquinoline [3] and derivatives [3 (b-d)].}

\subsection{Preparation of phenethylamine ${ }^{1}$}

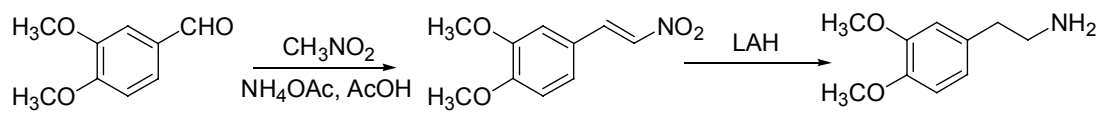

A mixture of verataldehyde $(4.00 \mathrm{~g}, 24.09 \mathrm{mmol})$, ammonium acetate $(1.85 \mathrm{~g}, 24.09 \mathrm{mmol})$, nitromethane ( $7.34 \mathrm{~g}, 120.45 \mathrm{mmol})$, and glacial AcOH was refluxed for $1.5 \mathrm{~h}$. After cooling to r.t., the crystalline product was filtered and recrystallized from $\mathrm{EtOH}$ to afford 1,2-dimethoxy-4(2-nitro-vinyl)-benzene yellow solid; Yield $=4.01 \mathrm{~g}(80 \%)$; mp: $148-150{ }^{\circ} \mathrm{C} ;{ }^{1} \mathrm{H} \mathrm{NMR}\left(\mathrm{CDCl}_{3}\right.$, $200 \mathrm{MHz}) \delta 7.96(\mathrm{~d}, 1 \mathrm{H}, J=13.6 \mathrm{~Hz}, \operatorname{ArH}), 7.53(\mathrm{~d}, 1 \mathrm{H}, J=13.6 \mathrm{~Hz}, \operatorname{ArH}), 7.18(\mathrm{dd}, 1 \mathrm{H}, J=$ 8.3, $1.8 \mathrm{~Hz}, \mathrm{ArH}), 7.01(\mathrm{~d}, 1 \mathrm{H}, J=1.7 \mathrm{~Hz}, \mathrm{ArH}), 6.92(\mathrm{~d}, 1 \mathrm{H}, J=8.3 \mathrm{~Hz}, \mathrm{ArH}), 3.94(\mathrm{~s}, 3 \mathrm{H}$, $\left.\mathrm{OCH}_{3}\right), 3.93\left(\mathrm{~s}, 3 \mathrm{H}, \mathrm{OCH}_{3}\right)$; IR (KBr) $v_{\max } 1627,1598,1580,1492,1340,1263$; MS (FAB) 210 for $[\mathrm{M}+1]^{+}$.

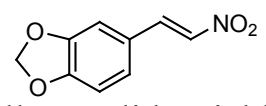

5-(2-Nitro-vinyl)-benzo[1,3]dioxole yellow solid; Yield = $3.93 \mathrm{~g}(85 \%)$; mp: $165-167{ }^{\circ} \mathrm{C} ;{ }^{1} \mathrm{H}$ $\operatorname{NMR}\left(\mathrm{CDCl}_{3}, 200 \mathrm{MHz}\right) \delta 7.91$ (s, 1H, ArH), 7.47 (s, 1H, ArH), 7.10-6.87 [m(o), 3H, ArH], 6.06 (s, 2H, ArH); IR (KBr) $v_{\max } 1628,1602,1495,1335,1271$; MS (FAB) 194 for $[\mathrm{M}+1]^{+}$. 
A solution of 1,2-Dimethoxy-4-(2-nitro-vinyl)-benzene (3.00 g, $15.54 \mathrm{mmol})$ in THF $(15 \mathrm{~mL})$ was added dropwise to a stirred solutionof $\mathrm{LiAlH}_{4}(1.18 \mathrm{~g}, 31.08 \mathrm{mmol})$ in THF $(45 \mathrm{~mL})$. The reaction mixture was refluxed for $3 \mathrm{~h}$ followed by addition of aq $\mathrm{KOH}(20 \%)$ to destroy the excess LiAlH4. The mixture was then extractedwith EtOAc $(3 \times 20 \mathrm{~mL})$, washed with brine $(3 \mathrm{~g} \times 20$ $\mathrm{mL})$, dried $\left(\mathrm{K}_{2} \mathrm{CO}_{3}\right)$, concentrated in vacuo. The oily residue was used directly in the next step (The resulting amines were instable, so consumed immediately).

\subsection{Preparation of $N$-[2-(3,4-dimethoxyphenyl)ethyl]-2-nitrobenzamide ${ }^{2}$}

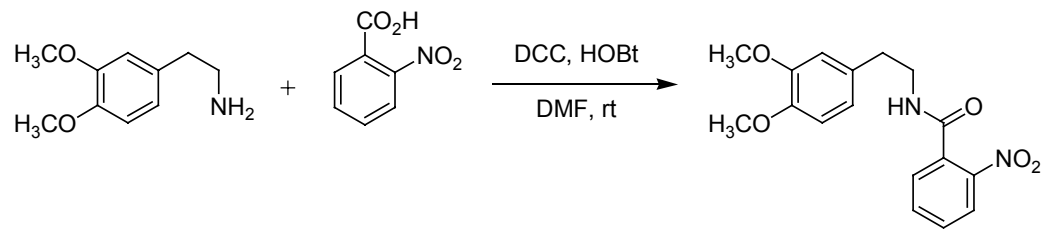

To a solution of $o$-nitrobenzoic acid $(0.58 \mathrm{~g}, 3.83 \mathrm{mmol})$ in $10 \mathrm{~mL}$ of DMF was added $\mathrm{HOBt}$ $(1.17 \mathrm{~g}, 7.66 \mathrm{mmol})$, followed by DCC $(1.58 \mathrm{~g}, 7.66 \mathrm{mmol})$. The reaction solution was stirred overnight. 2-(3,4-Dimethoxy-phenyl)-ethylamine $(0.69 \mathrm{~g}, 3.83 \mathrm{mmol})$ was added to the reaction solution, and the stirring was continued for another $12 \mathrm{~h}$. DCU was removed by filtration, and the filtrate was concentrated in vacuo. Column chromatography of the residue (in $\mathrm{CHCl}_{3}$ ) afforded $\mathrm{N}$ [2-(3,4-dimethoxyphenyl)ethyl]-2-nitrobenzamide. white solid; Yield $=1.20 \mathrm{~g}$ (95\%); mp: $141-142{ }^{\circ} \mathrm{C}\left(\operatorname{lit}^{3} 142-143{ }^{\circ} \mathrm{C}\right) ; \mathrm{t}_{\mathrm{R}}=14.72 \mathrm{~min} ; \mathrm{R}_{\mathrm{f}}=0.66\left(1: 19 \mathrm{MeOH}: \mathrm{CHCl}_{3}\right) ;{ }^{1} \mathrm{H} \mathrm{NMR}\left(\mathrm{CDCl}_{3}\right.$, $300 \mathrm{MHz}) \delta 8.03(\mathrm{~d}, 1 \mathrm{H}, J=7.8 \mathrm{~Hz}, \mathrm{ArH}), 7.63(\mathrm{t}, 1 \mathrm{H}, J=7.5 \mathrm{~Hz}, \mathrm{ArH}), 7.55$ (t, $1 \mathrm{H}, J=7.8 \mathrm{~Hz}$, ArH), 7.42 (d, 1H, J=7.2 Hz, ArH), 6.80 (s, 3H, ArH), 5.85 (brs, 1H, NH), 3.86 (s, 3H, $\mathrm{OCH}_{3}$ ), $3.85\left(\mathrm{~s}, 3 \mathrm{H}, \mathrm{OCH}_{3}\right), 3.74\left(\mathrm{q}, 2 \mathrm{H}, J=6.9 \mathrm{~Hz}, \mathrm{CH}_{2}\right), 2.92\left(\mathrm{t}, 2 \mathrm{H}, J=6.6 \mathrm{~Hz}, \mathrm{CH}_{2}\right)$; IR $(\mathrm{KBr}) v_{\max }$ $3301,1639,1555,1528,1359$; MS (FAB) 331 for $[\mathrm{M}+1]^{+}$.

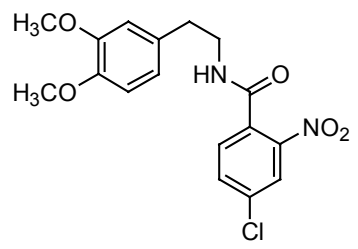

4-Chloro- $\boldsymbol{N}$-[2-(3,4-dimethoxyphenyl)ethyl]-2-nitrobenzamide white solid; Yield $=1.26 \mathrm{~g}$ (90 \%); mp: $164-166{ }^{\circ} \mathrm{C} ; \mathrm{t}_{\mathrm{R}}=16.01 \mathrm{~min} ; \mathrm{R}_{\mathrm{f}}=0.75\left(1: 19 \mathrm{MeOH}: \mathrm{CHCl}_{3}\right) ;{ }^{1} \mathrm{H} \mathrm{NMR}\left(\mathrm{CDCl}_{3}, 200 \mathrm{MHz}\right)$ $\delta 7.98(\mathrm{~d}, 1 \mathrm{H}, J=1.8 \mathrm{~Hz}, \operatorname{ArH}), 7.58(\mathrm{dd}, 1 \mathrm{H}, J=8.1,1.8 \mathrm{~Hz}, \operatorname{ArH}) 7.25(\mathrm{~d}, 1 \mathrm{H}, J=8.1 \mathrm{~Hz}$, $\mathrm{ArH}), 6.83-6.70[\mathrm{~m}(\mathrm{o}), 3 \mathrm{H}, \mathrm{ArH}], 6.09$ (brs, $1 \mathrm{H}, \mathrm{NH}), 3.85\left(\mathrm{~s}, 3 \mathrm{H}, \mathrm{OCH}_{3}\right), 3.84\left(\mathrm{~s}, 3 \mathrm{H}, \mathrm{OCH}_{3}\right)$, 3.68 (q, 2H, $\left.J=6.9 \mathrm{~Hz}, \mathrm{CH}_{2}\right), 2.89$ (t, $2 \mathrm{H}, J=6.8 \mathrm{~Hz}, \mathrm{CH}_{2}$ ); IR (KBr) v $v_{\max } 3432,1620,1553$, 1348 ; MS (FAB) 365 for $[\mathrm{M}+1]^{+}$.

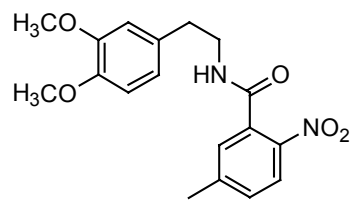

$\mathrm{N}$-[2-(3,4-Dimethoxyphenyl)ethyl]-5-methyl-2-nitrobenzamide white solid; Yield $=1.12 \mathrm{~g}$ (85 \%); mp: $150-152{ }^{\circ} \mathrm{C} ; \mathrm{t}_{\mathrm{R}}=15.23 \mathrm{~min} ; \mathrm{R}_{\mathrm{f}}=0.70$ (1:19 $\left.\mathrm{MeOH}: \mathrm{CHCl}_{3}\right) ;{ }^{1} \mathrm{H} \mathrm{NMR}\left(\mathrm{CDCl}_{3}, 200 \mathrm{MHz}\right)$ $\delta 7.96(\mathrm{~d}, 1 \mathrm{H}, J=8.4 \mathrm{~Hz}, \mathrm{ArH}), 7.32(\mathrm{~d}, 1 \mathrm{H}, J=8.2 \mathrm{~Hz}, \mathrm{ArH}), 7.19$ (s, 1H, ArH), 6.90-6.72 $[\mathrm{m}(\mathrm{o}), 3 \mathrm{H}, \mathrm{ArH}], 5.90(\mathrm{brs}, 1 \mathrm{H}, \mathrm{NH}), 3.86\left(\mathrm{~s}, 3 \mathrm{H}, \mathrm{OCH}_{3}\right), 3.85\left(\mathrm{~s}, 3 \mathrm{H}, \mathrm{OCH}_{3}\right), 3.72(\mathrm{q}, 2 \mathrm{H}, J=$ $\left.6.4 \mathrm{~Hz}, \mathrm{CH}_{2}\right), 2.91$ (t, $\left.2 \mathrm{H}, J=6.8 \mathrm{~Hz}, \mathrm{CH}_{2}\right), 2.42\left(\mathrm{~s}, 3 \mathrm{H}, \mathrm{CH}_{3}\right)$; IR $(\mathrm{KBr}) v_{\max } 3429,1625,1556$, 1352; MS (FAB) 345 for $[\mathrm{M}+1]^{+}$. 


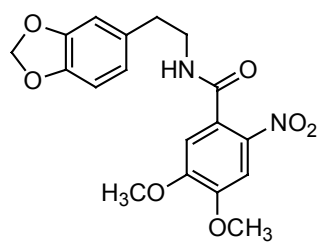

$\mathrm{N}$-(2-Benzo[1,3]dioxol-5-ylethyl)-4,5-dimethoxy-2-nitrobenzamide white solid; Yield = $1.16 \mathrm{~g}(81 \%) ; \mathrm{mp}: 168-170{ }^{\circ} \mathrm{C} ; \mathrm{t}_{\mathrm{R}}=14.95, \mathrm{R}_{\mathrm{f}}=0.71\left(1: 19 \mathrm{MeOH}: \mathrm{CHCl}_{3}\right) ;{ }^{1} \mathrm{H} \mathrm{NMR}\left(\mathrm{CDCl}_{3}, 200\right.$ MHz) $\delta 7.58(\mathrm{~s}, 1 \mathrm{H}, \mathrm{ArH}), 6.78(\mathrm{~s}, 1 \mathrm{H}, \mathrm{ArH}), 6.72-6.71[\mathrm{~m}(\mathrm{o}), 3 \mathrm{H}, \mathrm{ArH}], 5.91\left(\mathrm{~s}, 2 \mathrm{H}, \mathrm{CH}_{2}\right), 3.95$ (s, $\left.3 \mathrm{H}, \mathrm{OCH}_{3}\right), 3.94\left(\mathrm{~s}, 3 \mathrm{H}, \mathrm{OCH}_{3}\right), 3.65\left(\mathrm{q}, 2 \mathrm{H}, J=6.7 \mathrm{~Hz}, \mathrm{CH}_{2}\right), 2.87\left(\mathrm{t}, 2 \mathrm{H}, J=6.8 \mathrm{~Hz}, \mathrm{CH}_{2}\right)$; IR (KBr) $v_{\max } 3268,1634,1581,1520,1347,1219$; MS (FAB) 375 for [M+1] $]^{+}$.

\subsection{Preparation of 6,7-dimethoxy-1-(2-nitrophenyl)-3,4-dihydroisoquinoline [1a $]^{4}$}

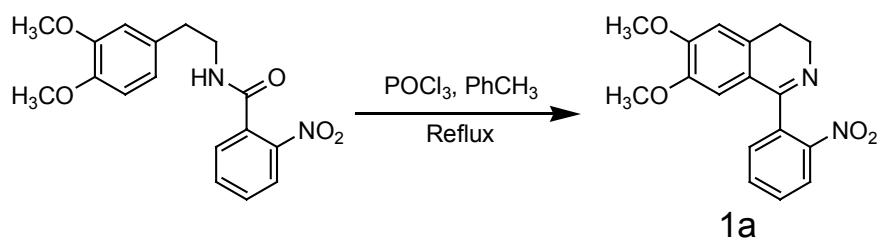

To a solution of $\mathrm{N}$-[2-(3,4-dimethoxyphenyl)ethyl]-2-nitrobenzamide (1.00 g, $3.02 \mathrm{mmol})$ in toluene $(30 \mathrm{~mL})$ at $\mathrm{rt}$ was added phosphorous oxychloride $(2.31 \mathrm{~g}, 15.1 \mathrm{mmol})$ dropwise over 5 min. The mixture was stirred at reflux for $3 \mathrm{~h}$ followed by cooling to rt. Toluene was removed under reduced pressure and solid residue was triturated with $10 \%$ aq. $\mathrm{NaOH}(20 \mathrm{~mL})$ to afford a suspension $(p \mathrm{H} \mathrm{8-9)}$. The suspension was extracted with EtOAc $(3 \times 50 \mathrm{~mL})$ The combined oranic extract were dried $\left(\mathrm{Na}_{2} \mathrm{SO}_{4}\right)$, filtered and concentrated under vacuum to give a crude solid. The crude was further purified by silica gel column chromatography with $40 \%$ EtOAc/hexane to give 6,7-dimethoxy-1-(2-nitrophenyl)-3,4-dihydroisoquinoline [1a] yellow solid; Yield $=0.80$ g (85\%); mp: $113-115^{\circ} \mathrm{C}\left(\right.$ lit $\left.^{5} 112-113{ }^{\circ} \mathrm{C}\right) ; \mathrm{t}_{\mathrm{R}}=11.80 \mathrm{~min} ; \mathrm{R}_{\mathrm{f}}=0.49(2: 3$ EtOAc:Hexane $) ;{ }^{1} \mathrm{H}$ NMR $\left(\mathrm{CDCl}_{3}, 300 \mathrm{MHz}\right) \delta 8.08(\mathrm{~d}, 1 \mathrm{H}, J=7.8 \mathrm{~Hz}, \mathrm{ArH}), 7.72(\mathrm{t}, 1 \mathrm{H}, J=6.9 \mathrm{~Hz}, \mathrm{ArH}), 7.59$ [t(o), 2H, J = 9.0 Hz, ArH), 6.77 (s, 1H, ArH), $6.33(\mathrm{~s}, 1 \mathrm{H}, \mathrm{ArH}), 3.93\left(\mathrm{~s}, 3 \mathrm{H}, \mathrm{OCH}_{3}\right), 3.85$ (t, 2H, $\left.J=9.0 \mathrm{~Hz}, \mathrm{CH}_{2}\right), 3.63\left(\mathrm{~s}, 3 \mathrm{H}, \mathrm{OCH}_{3}\right), 2.83\left(\mathrm{t}, 2 \mathrm{H}, J=9.0 \mathrm{~Hz}, \mathrm{CH}_{2}\right)$; IR $(\mathrm{KBr}) v_{\max } 1606,1568$, 1525, 1354; MS (FAB) 313 for $[\mathrm{M}+1]^{+}$.

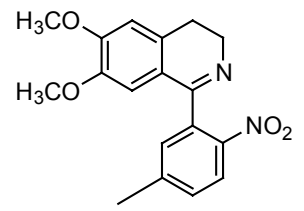

6,7-Dimethoxy-1-(5-methyl-2-nitrophenyl)-3,4-dihydroisoquinoline [1c] yellow solid; Yield = $0.74 \mathrm{~g}(76 \%)$; mp: $137-139{ }^{\circ} \mathrm{C} ; \mathrm{t}_{\mathrm{R}}=23.69 \mathrm{~min} ; \mathrm{R}_{\mathrm{f}}=0.50$ (2:3 EtOAc:Hexane); ${ }^{1} \mathrm{H} \mathrm{NMR}\left(\mathrm{CDCl}_{3}\right.$, $200 \mathrm{MHz}) \delta 8.00(\mathrm{~d}, 1 \mathrm{H}, J=8.8 \mathrm{~Hz}, \mathrm{ArH}), 7.37[\mathrm{~d}(\mathrm{o}), 2 \mathrm{H}, J=4.6 \mathrm{~Hz}, \mathrm{ArH}], 6.76(\mathrm{~s}, 1 \mathrm{H}, \mathrm{ArH})$, $6.31(\mathrm{~s}, 1 \mathrm{H}, \mathrm{ArH}), 3.92\left(\mathrm{~s}, 3 \mathrm{H}, \mathrm{OCH}_{3}\right), 3.86\left(\mathrm{t}, 2 \mathrm{H}, J=7.8 \mathrm{~Hz}, \mathrm{CH}_{2}\right), 3.63\left(\mathrm{~s}, 3 \mathrm{H}, \mathrm{OCH}_{3}\right), 2.83$ (t, $\left.2 \mathrm{H}, J=7.5 \mathrm{~Hz}, \mathrm{CH}_{2}\right), 2.49$ (s, 3H, $\mathrm{CH}_{3}$ ); IR (KBr) $v_{\max } 1602,1522,1351$; MS (FAB) 327 for $[\mathrm{M}+1]^{+}$. 


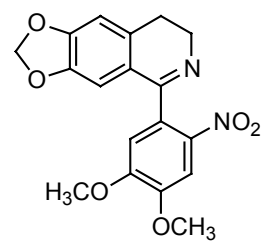

5-(4,5-Dimethoxy-2-nitrophenyl)-7,8-dihydro-[1,3]dioxolo[4,5-g]isoquinoline [1d] yellow oil; Yield $=0.86 \mathrm{~g}(80 \%) ; \mathrm{t}_{\mathrm{R}}=18.19 \mathrm{~min} ; \mathrm{R}_{\mathrm{f}}=0.48\left(2: 3\right.$ EtOAc:Hexane); ${ }^{1} \mathrm{H}$ NMR $\left(\mathrm{CDCl}_{3}, 200 \mathrm{MHz}\right) 7.72(\mathrm{~s}, 1 \mathrm{H}, \mathrm{ArH}), 6.93(\mathrm{~s}, 1 \mathrm{H}, \mathrm{ArH}), 6.75$ (s, $\left.1 \mathrm{H}, \mathrm{ArH}\right), 6.25(\mathrm{~s}, 1 \mathrm{H}, \mathrm{ArH})$, $5.93\left(\mathrm{~s}, 2 \mathrm{H}, \mathrm{CH}_{2}\right), 4.00\left(\mathrm{~s}, 3 \mathrm{H}, \mathrm{OCH}_{3}\right), 3.99\left(\mathrm{~s}, 3 \mathrm{H}, \mathrm{OCH}_{3}\right), 3.90\left(\mathrm{t}, 2 \mathrm{H}, J=7.8 \mathrm{~Hz}, \mathrm{CH}_{2}\right), 2.84$ (t, $2 \mathrm{H}, J=7.5 \mathrm{~Hz}, \mathrm{CH}_{2}$ ); IR (KBr) $v_{\max } 2931,1589,1519,1342,1265,1224$; MS (FAB) 357 for $[\mathrm{M}+1]^{+}$.

\subsection{Catalytic reduction of 1 a to 2-(6,7-dimethoxy-3,4-dihydroisoquinolin-1-yl)- phenylamine [2a]}<smiles>COc1cc2c(cc1OC)C(c1ccccc1[N+](=O)[O-])=NCC2</smiles>

$1 \mathrm{a}$

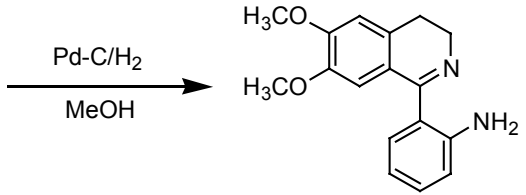

$2 a$

A mixture of compound $1 \mathrm{a}(0.20 \mathrm{~g}, 0.64 \mathrm{mmol})$ and $\mathrm{Pd}-\mathrm{C}(0.04 \mathrm{~g})$ in methanol $(10 \mathrm{~mL}) \mathrm{was}$ subjected to hydrogenation in the Parr assembly at $35 \mathrm{psi}$ at room temperature. The reaction was allowed to continue for $3 \mathrm{~h}$. thereafter, the catalyst was removed by vacuum filtering the reaction mixture through a Celite bed with methanol. The filtrate was evaporated to obtain an oily residue, which was taken up in EtOAc $(2 \times 20 \mathrm{~mL})$ and washed with water $(20 \mathrm{~mL})$. The organic layers were evaporated in vacuo to obtain the crude oil, which was recrystallized in ethanol to obtain 2(6,7-dimethoxy-3,4-dihydroisoquinolin-1-yl)phenylamine [2a] white solid; Yield $=0.17 \mathrm{~g}(95$ \%); mp: $154-156{ }^{\circ} \mathrm{C} ; \mathrm{t}_{\mathrm{R}}=15.38 \mathrm{~min} ; \mathrm{R}_{\mathrm{f}}=0.32$ (2:3 EtOAc:Hexane); ${ }^{1} \mathrm{H}$ NMR $\left(\mathrm{CDCl}_{3}, 300 \mathrm{MHz}\right)$

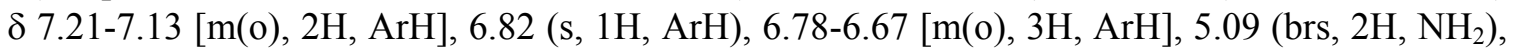
$3.94\left(\mathrm{~s}, 3 \mathrm{H}, \mathrm{OCH}_{3}\right), 3.81\left(\mathrm{t}, 2 \mathrm{H}, J=7.2 \mathrm{~Hz}, \mathrm{CH}_{2}\right), 3.72\left(\mathrm{~s}, 3 \mathrm{H}, \mathrm{OCH}_{3}\right) ; 2.69(\mathrm{t}, 2 \mathrm{H}, J=7.2 \mathrm{~Hz}$, $\mathrm{CH}_{2}$ ); IR (KBr) $v_{\max } 3423,1609$; MS (FAB) 283 for $[\mathrm{M}+1]^{+}$.

2.5 Basic $\mathrm{SnCl}_{2} .2 \mathrm{H}_{2} \mathrm{O}$ reduction of 1a to $\mathrm{N}$-[2-(6,7-dimethoxy-3,4-dihydroisoquinolin1-yl)phenyl]hydroxylamine ${ }^{6}$ [11a]

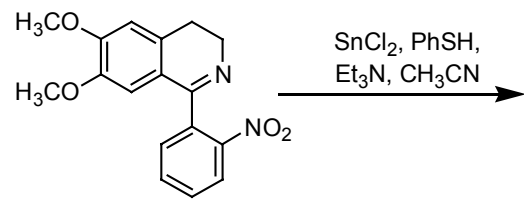

$1 \mathrm{a}$

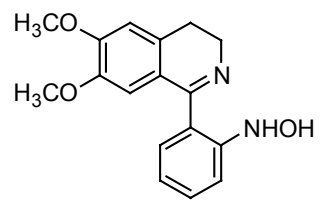

$11 \mathrm{a}$

To a solution of anhydrous $\mathrm{SnCl}_{2} .2 \mathrm{H}_{2} \mathrm{O}(0.22 \mathrm{~g}, 0.96 \mathrm{mmol})$ in $10 \mathrm{~mL}$ of $\mathrm{CH}_{3} \mathrm{CN}$, magnetically stirred at $\mathrm{rt}$ was sequentially added $\mathrm{PhSh}(0.29 \mathrm{~g}, 2.88 \mathrm{mmol})$ and $\mathrm{Et}_{3} \mathrm{~N}(0.44 \mathrm{~g}, 3.20 \mathrm{mmol})$. Then, $1 \mathrm{a}(0.20 \mathrm{~g}, 0.64 \mathrm{mmol})$ in $5 \mathrm{~mL}$ of $\mathrm{CH}_{3} \mathrm{CN}$ was added and stirred for $30 \mathrm{~min}$ at $\mathrm{rt}$. The reaction was concentrated in vacuum and the residue was separated by column chromatography (1:19 $\left.\mathrm{MeOH}: \mathrm{CHCl}_{3}\right)$ to afford $\mathbf{N}$-[2-(6,7-dimethoxy-3,4-dihydroisoquinolin-1yl)phenyl]hydroxylamine [11a] white solid; Yield $=0.17 \mathrm{~g}(95 \%) ; \mathrm{mp}: 149-151{ }^{\circ} \mathrm{C} ; \mathrm{t}_{\mathrm{R}}=$ $13.50 \mathrm{~min} ; \mathrm{R}_{\mathrm{f}}=0.45\left(1: 49 \mathrm{MeOH}: \mathrm{CHCl}_{3}\right) ;{ }^{1} \mathrm{H} \mathrm{NMR}\left(\mathrm{CDCl}_{3}, 300 \mathrm{MHz}\right) \delta 7.90(\mathrm{~d}, 1 \mathrm{H}, J=8.7 \mathrm{~Hz}$, $\mathrm{ArH}), 7.76(\mathrm{~d}, 1 \mathrm{H}, J=9.0 \mathrm{~Hz}, \mathrm{ArH}), 7.40(\mathrm{~s}, 1 \mathrm{H}, \mathrm{ArH}), 7.31(\mathrm{t}, 1 \mathrm{H}, J=8.4 \mathrm{~Hz}, \mathrm{ArH}), 7.19(\mathrm{t}, 1 \mathrm{H}$, $J=7.2 \mathrm{~Hz}, \mathrm{ArH}), 6.84(\mathrm{~s}, 1 \mathrm{H}, \mathrm{ArH}) 4.60\left(\mathrm{t}, 2 \mathrm{H}, J=6.0 \mathrm{~Hz}, \mathrm{CH}_{2}\right), 4.01\left(\mathrm{~s}, 3 \mathrm{H}, \mathrm{OCH}_{3}\right), 3.94(\mathrm{~s}$, 
$\left.3 \mathrm{H}, \mathrm{OCH}_{3}\right), 3.20\left(\mathrm{t}, 2 \mathrm{H}, J=6.0 \mathrm{~Hz}, \mathrm{CH}_{2}\right)$; IR $(\mathrm{KBr}) v_{\max } 3421,3325,2933,1606$; MS (FAB) 299 for $[\mathrm{M}+1]^{+}$.

\subsection{Cyclization of 11a to 3a in presence of TsCl.}

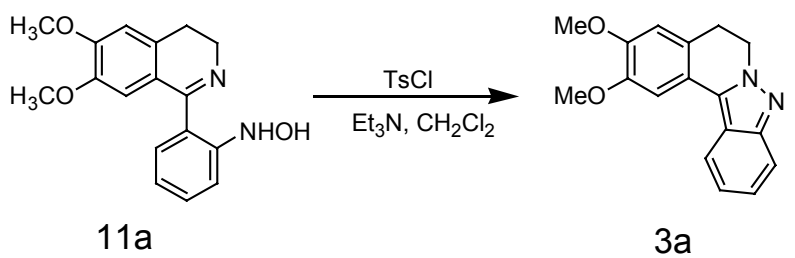

To a solution of 11a $(0.025 \mathrm{~g}, 0.08 \mathrm{mmol})$ and $\mathrm{Et}_{3} \mathrm{~N}(0.10 \mathrm{~g}, 0.10 \mathrm{mmol})$ in $2 \mathrm{~mL}$ of DCM was added tosyl chloride $(0.02 \mathrm{~g}, 0.10 \mathrm{mmol})$ solution in $1 \mathrm{~mL}$ DCM. The progress of the reaction was monitored by TLC and HPLC. After 20 min, solvent was evaporated and crude was extracted in EtOAc $(25 \mathrm{~mL})$. Then the EtOAc layer was washed with water $(2 \times 10 \mathrm{~mL})$, dried over $\mathrm{Na}_{2} \mathrm{SO}_{4}$, filtered and evaporated under reduced pressure to obtained 3a. The solid thus obtained was subjected to the characterization by ${ }^{1} \mathrm{H}$ NMR, ${ }^{13} \mathrm{C}$ NMR , HRMS (refer section 2.7) and X-ray crystallography (refer section 5).

\subsection{Intramolecular cyclization of 1a to 2,3-dimethoxy-5,6-dihydroindazolo[3,2- a]isoquinoline [3a]}

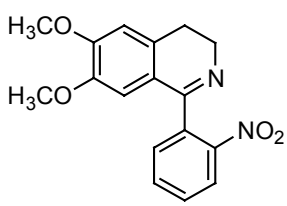

$1 \mathrm{a}$

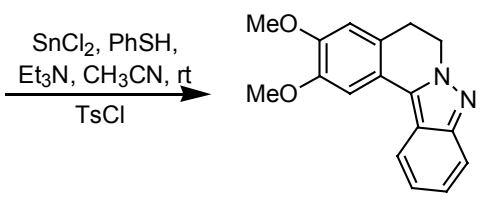

$3 a$

Triethyl amine $(0.45 \mathrm{~g}, 3.20 \mathrm{mmol})$ was added dropwise to a stirred solution of $\mathrm{SnCl}_{2} \cdot 2 \mathrm{H}_{2} \mathrm{O}(0.22$ $\mathrm{g}, 0.96 \mathrm{mmol})$ and $\mathrm{PhSH}(0.32 \mathrm{~g}, 2.90 \mathrm{mmol})$ in acetonitrile $(5 \mathrm{~mL})$ at room temperature to generate yellow precipitate over a period of $5 \mathrm{~min}$. Then $1 \mathrm{a}(0.180 \mathrm{~g}, 0.64 \mathrm{mmol})$ was transferred to the suspension and stirred at $\mathrm{rt}$ for 5 more min. After complete consumption of the nitro substrate on TLC, TsCl $(0.15 \mathrm{~g}, 0.83 \mathrm{mmol})$ was added to this solution and monitoring of the reaction was done on TLC. After completion of the reaction, the solvent was evaporated and the resulting residue was triturated with water $(10 \mathrm{~mL})$. The solid material was filtered and washed extensively with water. It was dried in a desiccator in vacuo and then again triturated with hexane. The solid was again filtered and washed extensively with hexane and then dried in vacuo. The solid so obtained was then digested with methanol and the insoluble material separated was removed by filtration. The filtrate was finally evaporated and the residue was crystallized from EtOAc-hexane to give 2,3-dimethoxy-5,6-dihydroindazolo[3,2-a]isoquinoline [3a]. white solid; Yield $=0.16 \mathrm{~g}(89 \%) ; \mathrm{mp}: 180-182{ }^{\circ} \mathrm{C} ; \mathrm{t}_{\mathrm{R}}=16.90 \mathrm{~min} ; \mathrm{R}_{\mathrm{f}}=0.63$ (2:3 EtOAc:Hexane); ${ }^{1} \mathrm{H}$ NMR $\left(\mathrm{CDCl}_{3}, 300 \mathrm{MHz}\right) \delta 7.96(\mathrm{~d}, 1 \mathrm{H}, J=8.4 \mathrm{~Hz}, \mathrm{ArH}), 7.73(\mathrm{~d}, 1 \mathrm{H}, J=8.7 \mathrm{~Hz}, \mathrm{ArH}), 7.48(\mathrm{~s}, 1 \mathrm{H}$, ArH), 7.32 (t, $1 \mathrm{H}, J=7.2 \mathrm{~Hz}, \mathrm{ArH}), 7.16(\mathrm{t}, 1 \mathrm{H}, J=7.5 \mathrm{~Hz}, \mathrm{ArH}), 6.85(\mathrm{~s}, 1 \mathrm{H}, \operatorname{ArH}), 4.62$ (t, 2H, $\left.J=6.9 \mathrm{~Hz}, \mathrm{CH}_{2}\right), 4.02\left(\mathrm{~s}, 3 \mathrm{H}, \mathrm{OCH}_{3}\right), 3.94\left(\mathrm{~s}, 3 \mathrm{H}, \mathrm{OCH}_{3}\right), 3.21\left(\mathrm{t}, 2 \mathrm{H}, J=6.9 \mathrm{~Hz}, \mathrm{CH}_{2}\right) ;{ }^{13} \mathrm{C}$ $\operatorname{NMR}\left(\mathrm{CDCl}_{3}, 50 \mathrm{MHz}\right) \delta 149.1,148.8,131.1,126.4,125.3,122.3,121.1,120.5,118.2,117.9$, 111.9, 107.7, 56.6, 56.5, 48.3, 29.1; IR (KBr) $v_{\max } 1603,1696,1217$; MS (FAB) 281 for $[\mathrm{M}+1]^{+}$; MS (HR EI) $\mathrm{m} / z$ calcd for $[\mathrm{M}]^{+} 280.12118$ found 280.12300 . 
2.8 Spectroscopic data of 3(b-d) Syntheses of these derivatives were commenced as per procedure described in the section 2.7 .

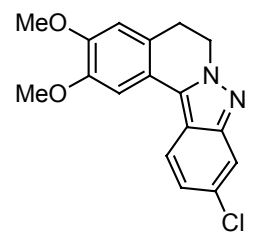

10-Chloro-2,3-dimethoxy-5,6-dihydroindazolo[3,2-a]isoquinoline [3b] pale yellow solid; Yield $=0.17 \mathrm{~g}(88 \%) ; \mathrm{mp}: 149-151{ }^{\circ} \mathrm{C} ; \mathrm{t}_{\mathrm{R}}=20.10 \mathrm{~min} ; \mathrm{R}_{\mathrm{f}}=0.75$ (2:3 EtOAc:Hexane); ${ }^{1} \mathrm{H}$ NMR $\left(\mathrm{CDCl}_{3}, 200 \mathrm{MHz}\right) \delta 7.87(\mathrm{~d}, 1 \mathrm{H}, J=8.9 \mathrm{~Hz}, \mathrm{ArH}), 7.69$ (d, $\left.1 \mathrm{H}, J=1.9 \mathrm{~Hz}, \mathrm{ArH}\right), 7.40(\mathrm{~s}, 1 \mathrm{H}$, ArH), $7.10(\mathrm{dd}, 1 \mathrm{H}, J=8.9,1.7 \mathrm{~Hz}, \mathrm{ArH}), 6.85(\mathrm{~s}, 1 \mathrm{H}, \mathrm{ArH}), 4.60\left(\mathrm{t}, 2 \mathrm{H}, J=6.9 \mathrm{~Hz}, \mathrm{CH}_{2}\right), 4.02$

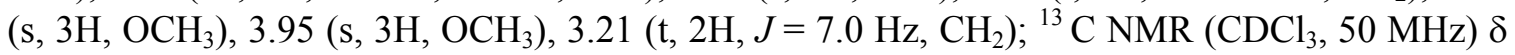
$149.5,149.1,148.9,132.2,131.7,125.5,123.5,121.7,120.5,117.2,116.3,112.0,107.8,56.7$, 56.4, 48.3, 29.1; IR (KBr) $v_{\max } 1589$; MS (FAB) 315 for [M+1] ${ }^{+}$; MS (HR EI) $\mathrm{m} / z$ calcd for $[\mathrm{M}]^{+}$ 314.08221 found 314.08275 .

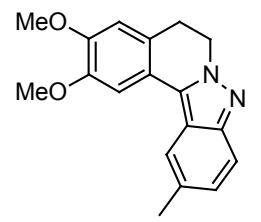

2,3-Dimethoxy-11-methyl-5,6-dihydroindazolo[3,2a]isoquinoline [3c] white solid; Yield = $0.18 \mathrm{~g}(90 \%) ; \mathrm{mp}: 152-154{ }^{\circ} \mathrm{C} ; \mathrm{t}_{\mathrm{R}}=18.50 \mathrm{~min} ; \mathrm{R}_{\mathrm{f}}=0.70$ (2:3 EtOAc:Hexane); ${ }^{1} \mathrm{H}$ NMR $\left(\mathrm{CDCl}_{3}\right.$, $200 \mathrm{MHz}) \delta 7.68(\mathrm{~s}, 1 \mathrm{H}, \operatorname{ArH}), 7.63(\mathrm{~d}, 1 \mathrm{H}, J=8.9 \mathrm{~Hz}, \operatorname{ArH}), 7.46(\mathrm{~s}, 1 \mathrm{H}, \operatorname{ArH}), 7.17(\mathrm{~d}, 1 \mathrm{H}, J=$ $8.8 \mathrm{~Hz}, \mathrm{ArH}), 6.85(\mathrm{~s}, 1 \mathrm{H}, \mathrm{ArH}), 4.59\left(\mathrm{t}, 2 \mathrm{H}, J=6.86 \mathrm{~Hz}, \mathrm{CH}_{2}\right), 4.04\left(\mathrm{~s}, 3 \mathrm{H}, \mathrm{OCH}_{3}\right), 3.95(\mathrm{~s}, 3 \mathrm{H}$, $\left.\mathrm{OCH}_{3}\right), 3.20\left(\mathrm{t}, 2 \mathrm{H}, J=7.04 \mathrm{~Hz}, \mathrm{CH}_{2}\right), 2.49\left(\mathrm{~s}, 3 \mathrm{H}, \mathrm{CH}_{3}\right) ;{ }^{13} \mathrm{C} \mathrm{NMR}\left(\mathrm{CDCl}_{3}, 50 \mathrm{MHz}\right) \delta 149.0$, $148.8,147.8,131.7,130.2,129.1,125.2,121.4,118.7,118.1,117.9,112.0,107.9,56.7,56.5$, 48.2, 29.2, 22.4; IR (KBr) $v_{\max } 1606,1539,1496,1218$; MS (FAB) 295 for [M+1] ; MS (HR EI) $\mathrm{m} / \mathrm{z}$ calcd for $[\mathrm{M}]^{+} 294.13683$ found 294.13635 .

\section{Experimental Procedure and Spectroscopic Data for 10-methyl-5,12- dihydro-6H-6a,7,12-triaza-indeno[1,2-a]fluorene $[14 \mathrm{c}]$ and derivatives [14a, 14b, 14d].}

\subsection{Preparation of $N$-[2-(1H-indol-3-yl)ethyl]-5-methyl-2-nitrobenzamide (12c)}

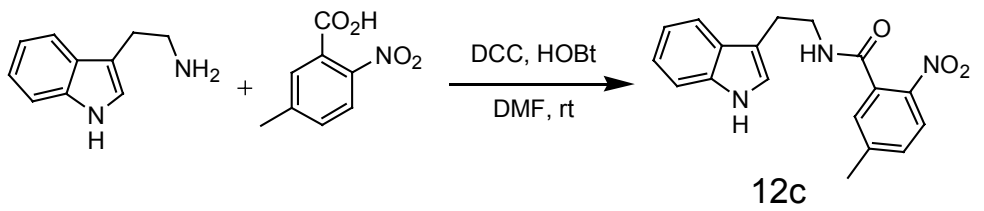

To a solution of 5-methyl-2-nitrobenzoic acid $(0.61 \mathrm{~g}, 3.83 \mathrm{mmol})$ in $10 \mathrm{~mL}$ of DMF was added HOBt $(1.17 \mathrm{~g}, 7.66 \mathrm{mmol})$, followed by DCC $(1.58 \mathrm{~g}, 7.66 \mathrm{mmol})$. The reaction solution was stirred overnight. tryptamine $(0.61 \mathrm{~g}, 3.83 \mathrm{mmol})$ was added to the reaction solution, and the stirring was continued for another $12 \mathrm{~h}$. DCU was removed by filtration, and the filtrate was concentrated in vacuo. Column chromatography of the residue (in $\left.\mathrm{CH}_{3} \mathrm{Cl}\right) \mathbf{N}$-[2-(1H -indol-3yl)ethyl]-5-methyl-2-nitrobenzamide [12c]. white solid; Yield = $1.08 \mathrm{~g}$ (91\%); mp: 146-148 ${ }^{\circ} \mathrm{C} ; \mathrm{t}_{\mathrm{R}}=16.85 \mathrm{~min} ; \mathrm{R}_{\mathrm{f}}=0.52\left(1: 19 \mathrm{MeOH}: \mathrm{CHCl}_{3}\right) ;{ }^{1} \mathrm{H} \mathrm{NMR}\left(\mathrm{CDCl}_{3}, 200 \mathrm{MHz}\right) \delta 8.12$ (brs, $1 \mathrm{H}$, 
$\mathrm{NH}), 7.93(\mathrm{~d}, 1 \mathrm{H}, J=8.4 \mathrm{~Hz}, \mathrm{ArH}), 7.63(\mathrm{~d}, 1 \mathrm{H}, J=7.4 \mathrm{~Hz}, \mathrm{ArH}), 7.34$ (t, $1 \mathrm{H}, J=7.6 \mathrm{~Hz}, \mathrm{ArH})$, 7.26-7.07 [m(overlapped with $\left.\mathrm{CDCl}_{3}\right), 5 \mathrm{H}, \mathrm{ArH}$ ], 5.81(brs, $\left.1 \mathrm{H}, \mathrm{NH}\right), 3.82$ (q, 2H, $J=6.4 \mathrm{~Hz}$, $\mathrm{CH}_{2}$ ), 3.12 (t, $\left.2 \mathrm{H}, J=6.5 \mathrm{~Hz}, \mathrm{CH}_{2}\right), 2.38\left(\mathrm{~s}, 3 \mathrm{H}, \mathrm{CH}_{3}\right)$; IR $(\mathrm{KBr}) v_{\max } 3428,3298,1637,1589$, 1548, 1521, 1353; MS (FAB) 324 for $[\mathrm{M}+1]^{+}$.

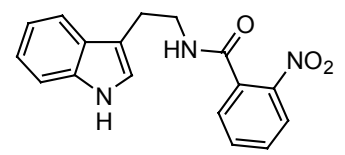

$\boldsymbol{N}$-[2-(1H-Indol-3-yl)ethyl]-2-nitrobenzamide [12a]. White solid; Yield = $1.12 \mathrm{~g}(95 \%)$; mp: $177-178{ }^{\circ} \mathrm{C} ; \mathrm{t}_{\mathrm{R}}=16.12 \mathrm{~min} ; \mathrm{R}_{\mathrm{f}}=0.58\left(1: 19 \mathrm{MeOH}: \mathrm{CHCl}_{3}\right) ;{ }^{1} \mathrm{H} \mathrm{NMR}\left(\mathrm{CDCl}_{3}, 200 \mathrm{MHz}\right) \delta$ 8.19 (brs, 1H, NH), 7.97 (d, $1 \mathrm{H}, J=8.4 \mathrm{~Hz}, \mathrm{ArH}), 7.62-7.52$ [m (o), 3H, ArH], 7.37-7.32 [m (o), 2H, ArH], 7.14-7.09 [m (o), 3H, ArH], 5.95 (brs, 1H, NH), 3.79 (t, $2 \mathrm{H}, J=5.3 \mathrm{~Hz}, \mathrm{CH}_{2}$ ), 3.12 (t, $2 \mathrm{H}, J=5.1 \mathrm{~Hz}, \mathrm{CH}_{2}$ ); IR (KBr) $v_{\max } 3410,1597,1526,1350$; MS (FAB) 310 for $[\mathrm{M}+1]^{+}$.

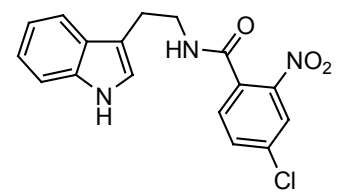

4-Chloro- $\boldsymbol{N}$-[2-(1H-indol-3-yl)ethyl]-2-nitrobenzamide [12b]. White solid; Yield $=1.16$ g (89\%); mp: $148-150{ }^{\circ} \mathrm{C} ; \mathrm{t}_{\mathrm{R}}=16.32 \mathrm{~min} ; \mathrm{R}_{\mathrm{f}}=0.61\left(1: 19 \mathrm{MeOH}: \mathrm{CHCl}_{3}\right) ;{ }^{1} \mathrm{H} \mathrm{NMR}\left(\mathrm{CDCl}_{3}, 200\right.$ $\mathrm{MHz}) \delta 8.06$ (brs, 1H, NH), 8.00 (s, 1H, ArH), 7.65-7.54 [m (o), 3H, ArH], 7.40-7.12 [m (overlapped with $\mathrm{CDCl}_{3}$ ), 4H, $\mathrm{ArH}$ ], 5.81 (brs, $\left.1 \mathrm{H}, \mathrm{NH}\right), 3.83$ (q, $2 \mathrm{H}, J=6.3 \mathrm{~Hz}, \mathrm{CH}_{2}$ ), 3.12 (t, $\left.2 \mathrm{H}, J=6.6 \mathrm{~Hz}, \mathrm{CH}_{2}\right)$; IR (KBr) $v_{\max } 3421,3285,1653,1538,1351,1219,770 ; \mathrm{MS}(\mathrm{FAB}) 344$ for $[\mathrm{M}+1]^{+}$.

\subsection{Preparation of 1-(5-methyl-2-nitro-phenyl)-4,9-dihydro-3H- $\beta$-carboline [13c].}
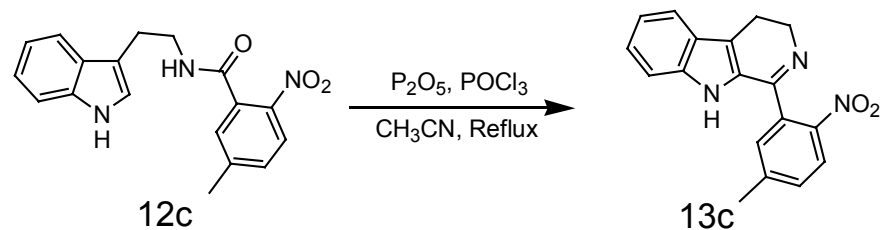

Phosphorous oxychloride $(2.48 \mathrm{~g}, 16.18 \mathrm{mmol})$ was added dropwise to a suspension of phosphorus pentoxide $(3.22 \mathrm{~g}, 22.68 \mathrm{mmol})$ in a solution of amide $12 \mathrm{c}(1.00 \mathrm{~g}, 3.24 \mathrm{mmol})$ in anhydrous toluene $(25 \mathrm{ml})$. After complete addition, the reaction mixture was heated under reflux for 2 hours and was then quenched by the addition of crushed ice $(25 \mathrm{ml})$. The aqueous residue was made basic by the addition of sodium hydroxide $(15 \% \mathrm{w} / \mathrm{v})$ solution and the product was then extracted into ethyl acetate $(3 \times 25 \mathrm{ml})$. The organic layer was washed with water, dried $\left(\mathrm{NaSO}_{4}\right)$ and evaporated under reduced pressure to obtained crude oil. The product was purified over silica (90: 10 hexane-ethyl acetate) to give 1-(5-methyl-2-nitrophenyl)-4,9-dihydro3H- $\beta$-carboline [13c] yellow solid; Yield $=0.66 \mathrm{~g}(70 \%) ; \mathrm{mp}: 189-191{ }^{\circ} \mathrm{C} ; \mathrm{t}_{\mathrm{R}}=12.77 \mathrm{~min} ; \mathrm{R}_{\mathrm{f}}=$ $0.45\left(1: 19 \mathrm{MeOH}: \mathrm{CHCl}_{3}\right) ;{ }^{1} \mathrm{H} \mathrm{NMR}\left(\mathrm{CDCl}_{3}, 200 \mathrm{MHz}\right) \delta 8.03$ [d(o), $\left.2 \mathrm{H}, J=8.2 \mathrm{~Hz}, \mathrm{ArH}\right], 7.63$ $(\mathrm{d}, 1 \mathrm{H}, J=7.8 \mathrm{~Hz}, \mathrm{ArH}), 7.39-7.11$ [m(overlapped with $\left.\left.\mathrm{CDCl}_{3}\right), 4 \mathrm{H}, \mathrm{ArH}\right], 4.03$ (t, $2 \mathrm{H}, J=8.2$ $\left.\mathrm{Hz}, \mathrm{CH}_{2}\right), 3.02\left(\mathrm{t}, 2 \mathrm{H}, J=8.8 \mathrm{~Hz}, \mathrm{CH}_{2}\right), 2.45\left(\mathrm{~s}, 3 \mathrm{H}, \mathrm{CH}_{3}\right)$; IR $(\mathrm{KBr}) v_{\max } 3423,3103,2933,1592$, 1517, 1342; MS (FAB) 306 for $[\mathrm{M}+1]^{+}$. 


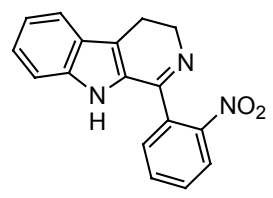

1-(2-Nitrophenyl)-4,9-dihydro-3H- $\beta$-carboline [13a] yellow solid; Yield $=0.65 \mathrm{~g}(69 \%)$; mp: $145-147{ }^{\circ} \mathrm{C} ; \mathrm{t}_{\mathrm{R}}=14.72 \mathrm{~min} ; \mathrm{R}_{\mathrm{f}}=0.48\left(1: 19 \mathrm{MeOH}: \mathrm{CHCl}_{3}\right) ;{ }^{1} \mathrm{H} \mathrm{NMR}\left(\mathrm{CDCl}_{3}, 200 \mathrm{MHz}\right) \delta$ $8.10[\mathrm{~d}, 1 \mathrm{H}, J=8.0 \mathrm{~Hz}, \mathrm{ArH}], 7.85-7.55[\mathrm{~m}(\mathrm{o}), 5 \mathrm{H}, \mathrm{ArH}], 7.36-7.15$ [m(overlapped with $\mathrm{CDCl}_{3}$ ), $2 \mathrm{H}, \mathrm{ArH}], 4.05\left(\mathrm{t}, 2 \mathrm{H}, J=8.8 \mathrm{~Hz}, \mathrm{CH}_{2}\right), 3.02\left(\mathrm{t}, 2 \mathrm{H}, J=8.3 \mathrm{~Hz}, \mathrm{CH}_{2}\right)$; IR (KBr) $v_{\max } 3292,1643$, $1600,1533,1355$; MS (FAB) 292 for $[\mathrm{M}+1]^{+}$.

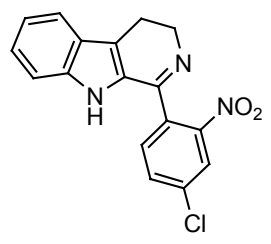

1-(4-Chloro-2-nitro-phenyl)-4,9-dihydro-3H- $\beta$-carboline [13b] yellow solid; Yield = $0.73 \mathrm{~g}(70 \%) ; \mathrm{mp}: 144-146{ }^{\circ} \mathrm{C} ; \mathrm{t}_{\mathrm{R}}=13.12 \mathrm{~min} ; \mathrm{R}_{\mathrm{f}}=0.51\left(1: 19 \mathrm{MeOH}: \mathrm{CHCl}_{3}\right) ;{ }^{1} \mathrm{H} \mathrm{NMR}\left(\mathrm{CDCl}_{3}\right.$, $200 \mathrm{MHz}) \delta 8.07$ (brs, $1 \mathrm{H}, \mathrm{NH}), 8.05$ (s, 1H, ArH), 7.68-7.58 [m(o), 3H, ArH], 7.50 (d, 1H, $J=$ $8.2 \mathrm{~Hz}, \mathrm{ArH}$ ), 7.25-7.12 [m(overlapped with $\mathrm{CDCl}_{3}$ ), $2 \mathrm{H}, \mathrm{ArH}$ ], 4.03 (t, $2 \mathrm{H}, J=8.3 \mathrm{~Hz}, \mathrm{CH}_{2}$ ), $3.00\left(\mathrm{t}, 2 \mathrm{H}, J=8.8 \mathrm{~Hz}, \mathrm{CH}_{2}\right.$ ); IR (KBr) $v_{\max } 3406,1599$, 1535, 1352; MS (FAB) 326 for $[\mathrm{M}+1]^{+}$.

\subsection{Intramolecular cyclization of $13 \mathrm{c}$ to 10 -methyl-5,12-dihydro-6H-6a,7,12-triaza- indeno[1,2-a]fluorene [14c]}
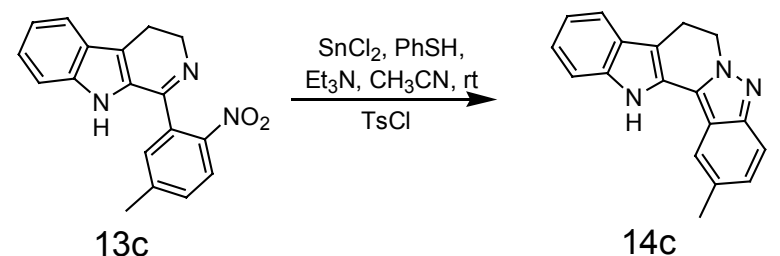

Triethyl amine $(0.45 \mathrm{~g}, 3.20 \mathrm{mmol})$ was added dropwise to a stirred solution of $\mathrm{SnCl}_{2} \cdot 2 \mathrm{H}_{2} \mathrm{O}(0.22$ $\mathrm{g}, 0.96 \mathrm{mmol})$ and $\mathrm{PhSH}(0.32 \mathrm{~g}, 2.90 \mathrm{mmol})$ in acetonitrile $(5 \mathrm{~mL})$ at room temperature to generate yellow precipitate over a period of $5 \mathrm{~min}$. Then $13 \mathrm{c}(0.18 \mathrm{~g}, 0.64 \mathrm{mmol})$ was transferred to the suspension and stirred at $\mathrm{rt}$ for 5 more min. After complete consumption of the nitro substrate on TLC, TsCl $(0.15 \mathrm{~g}, 0.83 \mathrm{mmol})$ was added to this solution and monitoring of the reaction was done on TLC. After completion of the reaction, the solvent was evaporated and the reaction was further proceeded as desribed in section 2.5 (supporting information) to give 10methyl-5,12-dihydro-6H-6a,7,12-triaza-indeno[1,2-a]fluorene [14c] pale yellow solid; Yield = $0.15 \mathrm{~g}(88 \%) ; \mathrm{mp}: 254-257{ }^{\circ} \mathrm{C} ; \mathrm{t}_{\mathrm{R}}=20.10 \mathrm{~min} ; \mathrm{R}_{\mathrm{f}}=0.62$ (2:3 EtOAc:Hexane); ${ }^{1} \mathrm{H}$ NMR $\left(\mathrm{CDCl}_{3}\right.$, $200 \mathrm{MHz}) \delta 8.72$ (brs, $1 \mathrm{H}, \mathrm{NH}), 7.66-7.58[\mathrm{~m}(\mathrm{o}), 3 \mathrm{H}, \mathrm{ArH}], 7.44$ (d, $1 \mathrm{H}, J=8.9 \mathrm{~Hz}, \mathrm{ArH}), 7.25-$ 7.17 [m(overlapped with $\left.\mathrm{CDCl}_{3}\right), 3 \mathrm{H}, \mathrm{ArH}$ ] $, 4.71\left(\mathrm{t}, 2 \mathrm{H}, J=7.5 \mathrm{~Hz}, \mathrm{CH}_{2}\right), 3.33(\mathrm{t}, 2 \mathrm{H}, J=7.5$ $\left.\mathrm{Hz}, \mathrm{CH}_{2}\right), 2.44\left(\mathrm{~s}, 3 \mathrm{H}, \mathrm{CH}_{3}\right) ;{ }^{13} \mathrm{C}$ NMR (DMSO-d $\left.6,50 \mathrm{MHz}\right) \delta 146.7,138.0,130.8,128.9,126.4$, 126.1, 125.9, 122.3, 120.0, 118.8, 118.7, 117.4, 116.6, 112.1, 107.2, 49.0, 21.8, 20.6; IR (KBr) $v_{\max } 2920,1594,1350$; MS (FAB) 274 for $[\mathrm{M}+1]^{+}$; MS (HR EI) $\mathrm{m} / z$ calcd 273.12660 for $[\mathrm{M}]^{+}$ found 273.12655 . 
3.4 Spectroscopic data of [14a, 14b, 14d] Syntheses of these derivatives were commenced as per procedure described in the section 3.3.

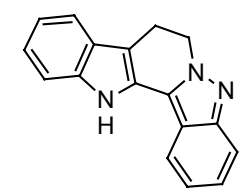

5,12-Dihydro-6H-6a,7,12-triazaindeno[1,2a]fluorene [14a]. pale yellow solid; Yield $=0.146 \mathrm{~g}$ (88 \%); mp: $256-258{ }^{\circ} \mathrm{C} ; \mathrm{t}_{\mathrm{R}}=19.40 \mathrm{~min} ; \mathrm{R}_{\mathrm{f}}=0.52$ (2:3 EtOAc:Hexane); ${ }^{1} \mathrm{H}$ NMR $\left(\mathrm{CDCl}_{3}, 200\right.$ MHz) $\delta 8.88(\mathrm{~s}, 1 \mathrm{H}, \mathrm{NH}), 7.86(\mathrm{~d}, 1 \mathrm{H}, J=7.3 \mathrm{~Hz}, \operatorname{ArH}), 7.74(\mathrm{~d}, 1 \mathrm{H}, J=7.6 \mathrm{~Hz}, \operatorname{ArH}), 7.57(\mathrm{~d}$, $1 \mathrm{H}, J=6.9 \mathrm{~Hz}, \mathrm{ArH}), 7.47$ (d, 1H, $J=6.7 \mathrm{~Hz}, \mathrm{ArH}), 7.31-7.17$ [m (o), 4H, ArH], 4.75 (t, 2H, $J=$ $\left.7.6 \mathrm{~Hz}, \mathrm{CH}_{2}\right), 3.35\left(\mathrm{t}, 2 \mathrm{H}, J=7.6 \mathrm{~Hz}, \mathrm{CH}_{2}\right) ;{ }^{13} \mathrm{C}$ NMR (DMSO- $\left.d_{6}, 50 \mathrm{MHz}\right) \delta 147.7,138.1$, 126.9, 126.2, 126.1, 122.5, 121.8, 120.8, 120.1, 118.8, 117.6, 116.4, 112.2, 107.6, 49.1, 20.6; IR (KBr) $v_{\max } 3191,1349,1311$; MS (FAB) 260 for $[\mathrm{M}+1]^{+}$; MS (HR EI) $\mathrm{m} / z$ calcd 259.11095 for $[\mathrm{M}]^{+}$found 259.11095.

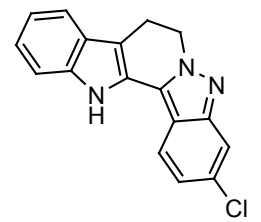

9-Chloro-5,12-dihydro-6H-6a,7,12-triazaindeno[1,2-a]fluorene [14b] pale yellow solid; Yield $=0.17 \mathrm{~g}(90 \%) ; \mathrm{mp}: 275-277{ }^{\circ} \mathrm{C} ; \mathrm{t}_{\mathrm{R}}=21.16 \mathrm{~min} ; \mathrm{R}_{\mathrm{f}}=0.68$ (2:3 EtOAc:Hexane); ${ }^{1} \mathrm{H}$ NMR $\left(\mathrm{CDCl}_{3}+20 \mu \mathrm{L}\right.$ DMSO- $\left.d_{6}, 200 \mathrm{MHz}\right) \delta 8.22(\mathrm{~d}, 1 \mathrm{H}, J=8.9 \mathrm{~Hz}, \mathrm{ArH}), 7.64(\mathrm{~s}, 1 \mathrm{H}, \mathrm{ArH}), 7.57-$ $7.42[\mathrm{~m} \mathrm{(o)}, 2 \mathrm{H}, \mathrm{ArH}], 7.20-7.04$ [m (o), 3H, ArH], 4.71 (t, $\left.2 \mathrm{H}, J=7.6 \mathrm{~Hz}, \mathrm{CH}_{2}\right), 3.36$ (t, $2 \mathrm{H}, J=$ $7.6 \mathrm{~Hz}, \mathrm{CH}_{2}$ ); IR (KBr) $v_{\max } 3450,1593,1349$; MS (FAB) 294 for [M+1] ; MS (HR EI) $\mathrm{m} / \mathrm{z}$ calcd 293.07197 for $[\mathrm{M}]^{+}$found 293.07265. 


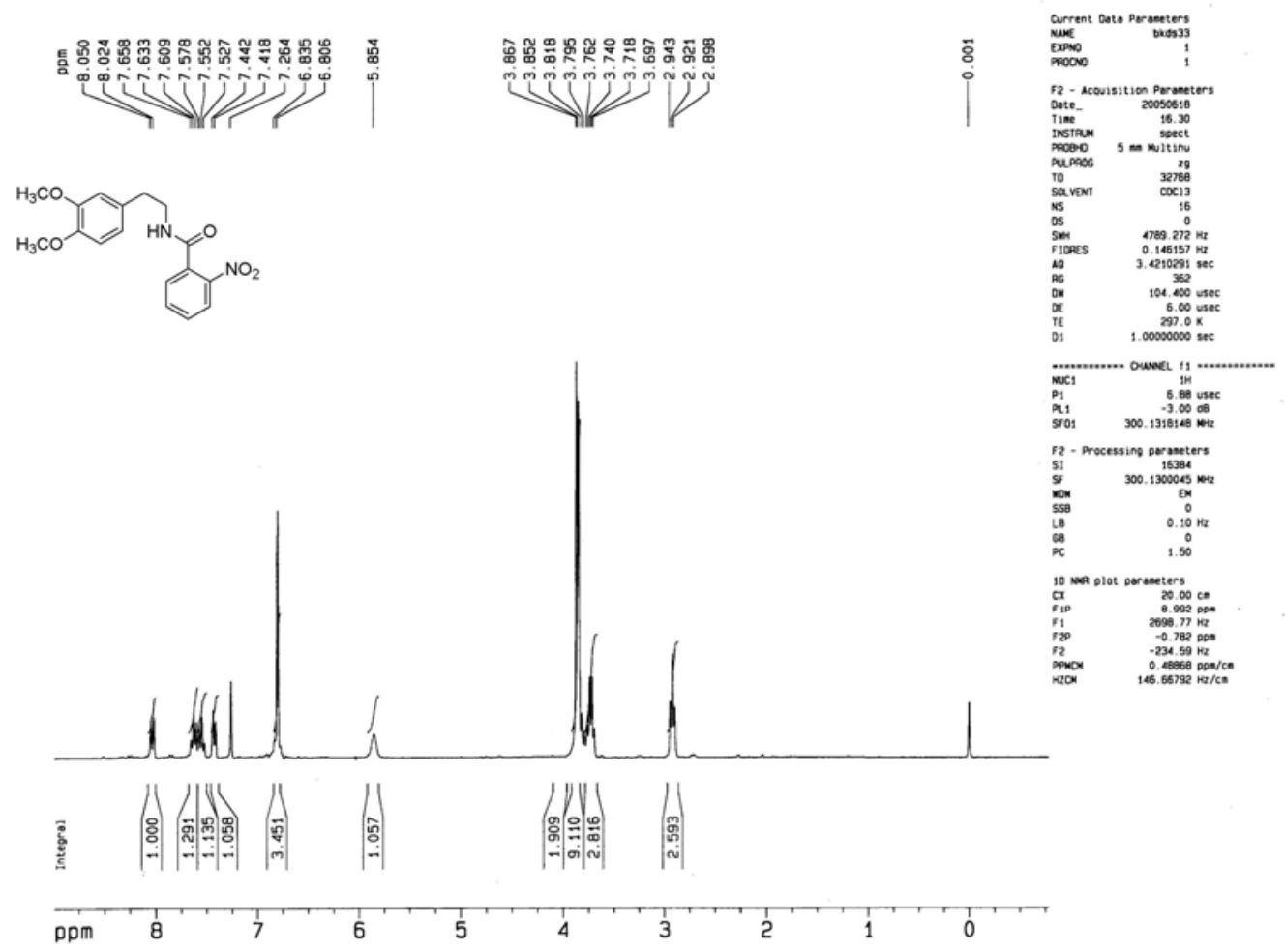

Fig 1: ${ }^{1} \mathrm{H}$ NMR of N-[2-(3,4-dimethoxyphenyl)ethyl]-2-nitrobenzamide

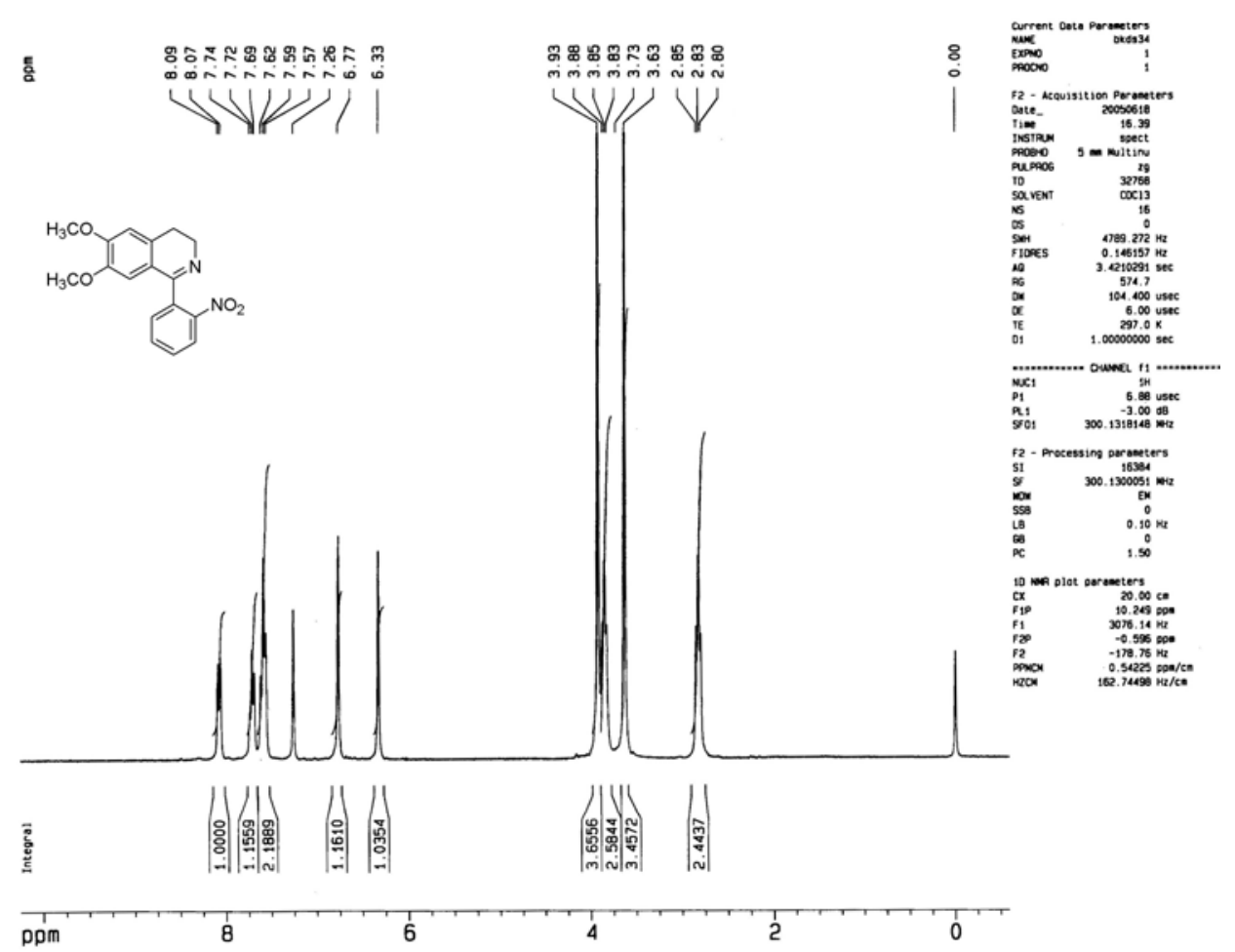

Fig 2: ${ }^{1} \mathrm{H}$ NMR of 6,7-Dimethoxy-1-(2-nitrophenyl)-3,4-dihydroisoquinoline [1a] 


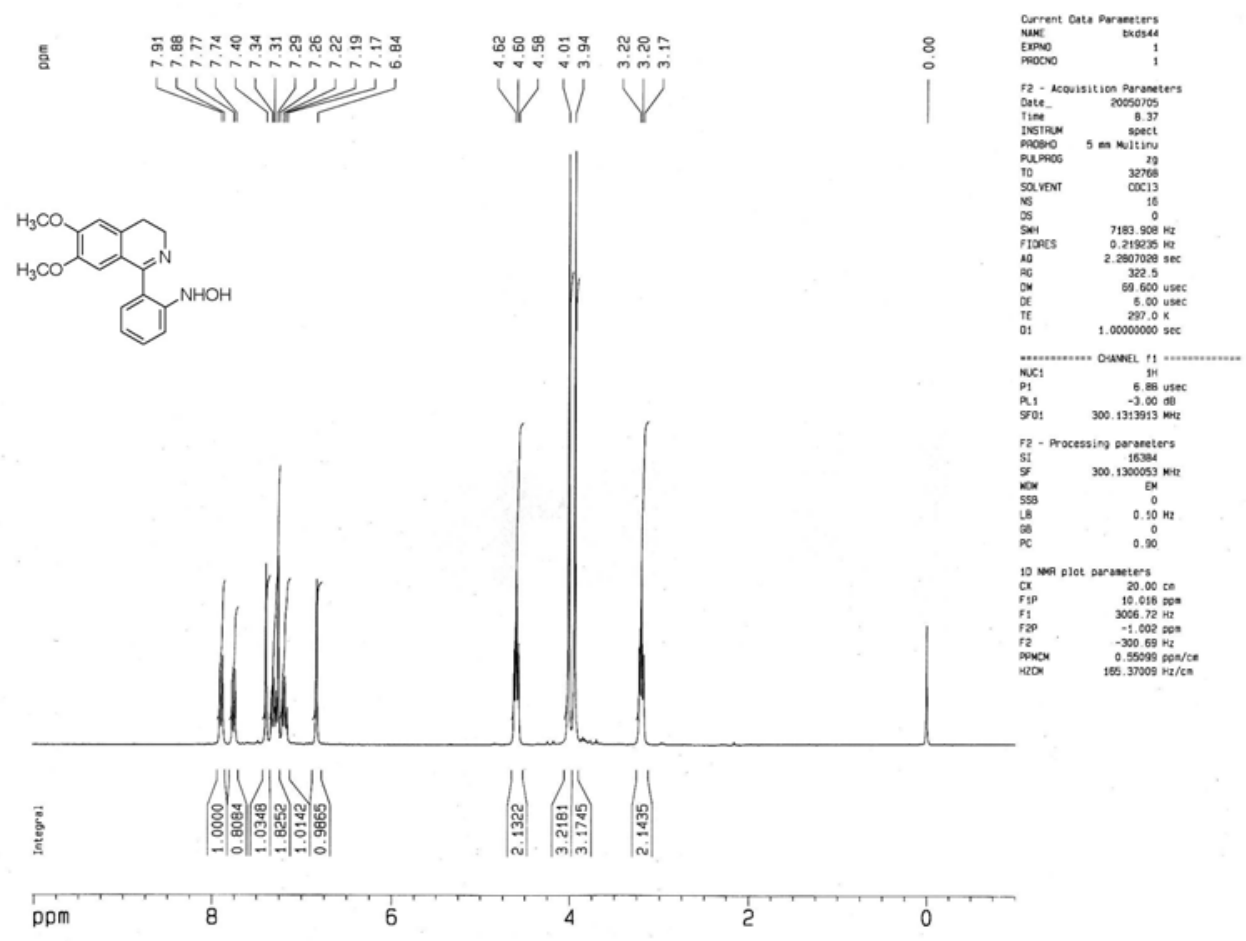

Fig 3: ${ }^{1} \mathrm{H} \quad \mathrm{NMR} \quad$ of $\quad \mathrm{N}$-[2-(6,7-Dimethoxy-3,4-dihydroisoquinolin-1-yl)phenyl]hydroxylamine [11a]

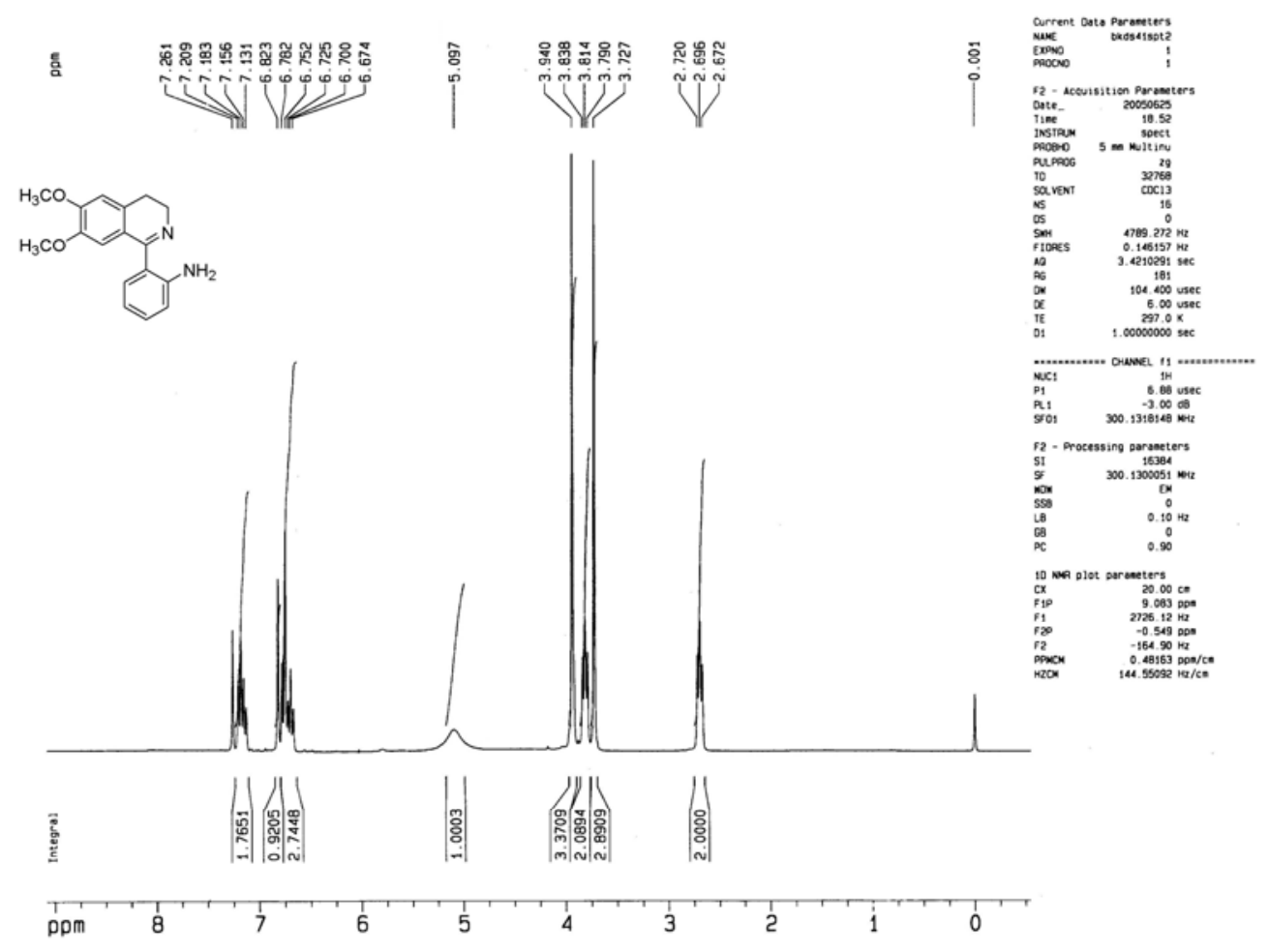

Fig 4: ${ }^{1} \mathrm{H}$ NMR of 2-(6,7-Dimethoxy-3,4-dihydroisoquinolin-1-yl)-phenylamine [2a] 


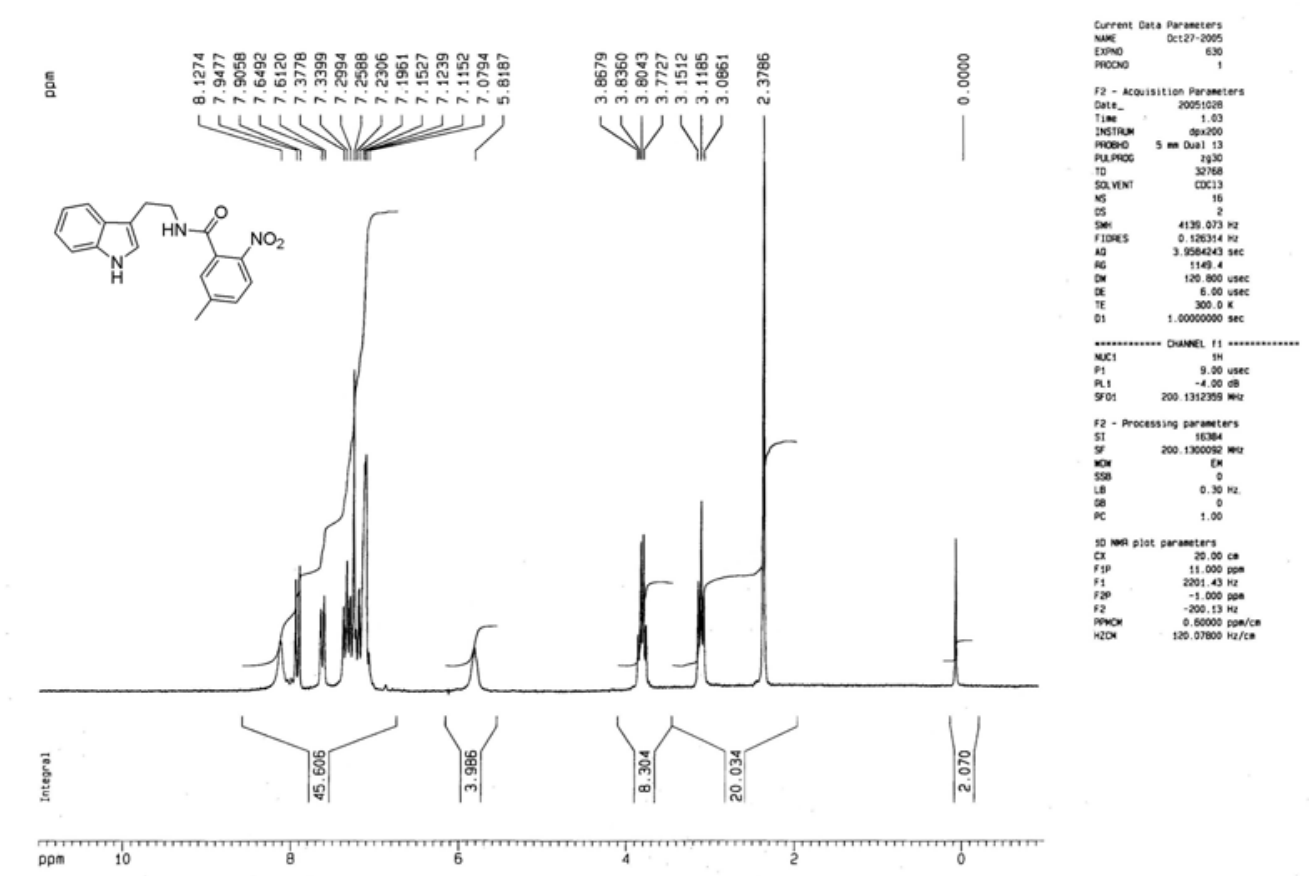

Fig 5: ${ }^{1} \mathrm{H}$ NMR of N-[2-(1H-Indol-3-yl)-ethyl]-5-methyl-2-nitro-benzamide [12c]

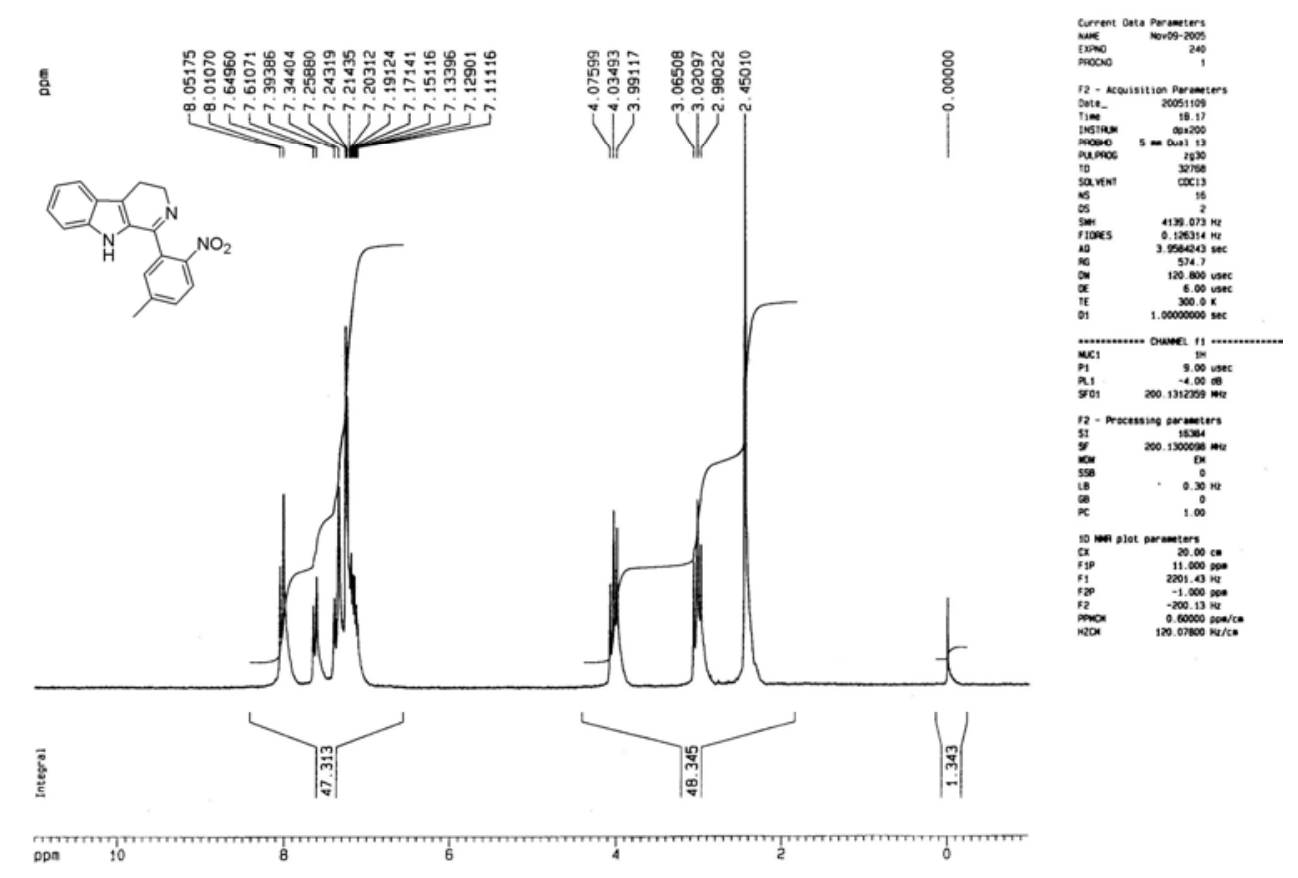

Fig 6: ${ }^{1} \mathrm{H}$ NMR of 1-(5-Methyl-2-nitro-phenyl)-4,9-dihydro-3H- $\beta$-carboline [13c] 


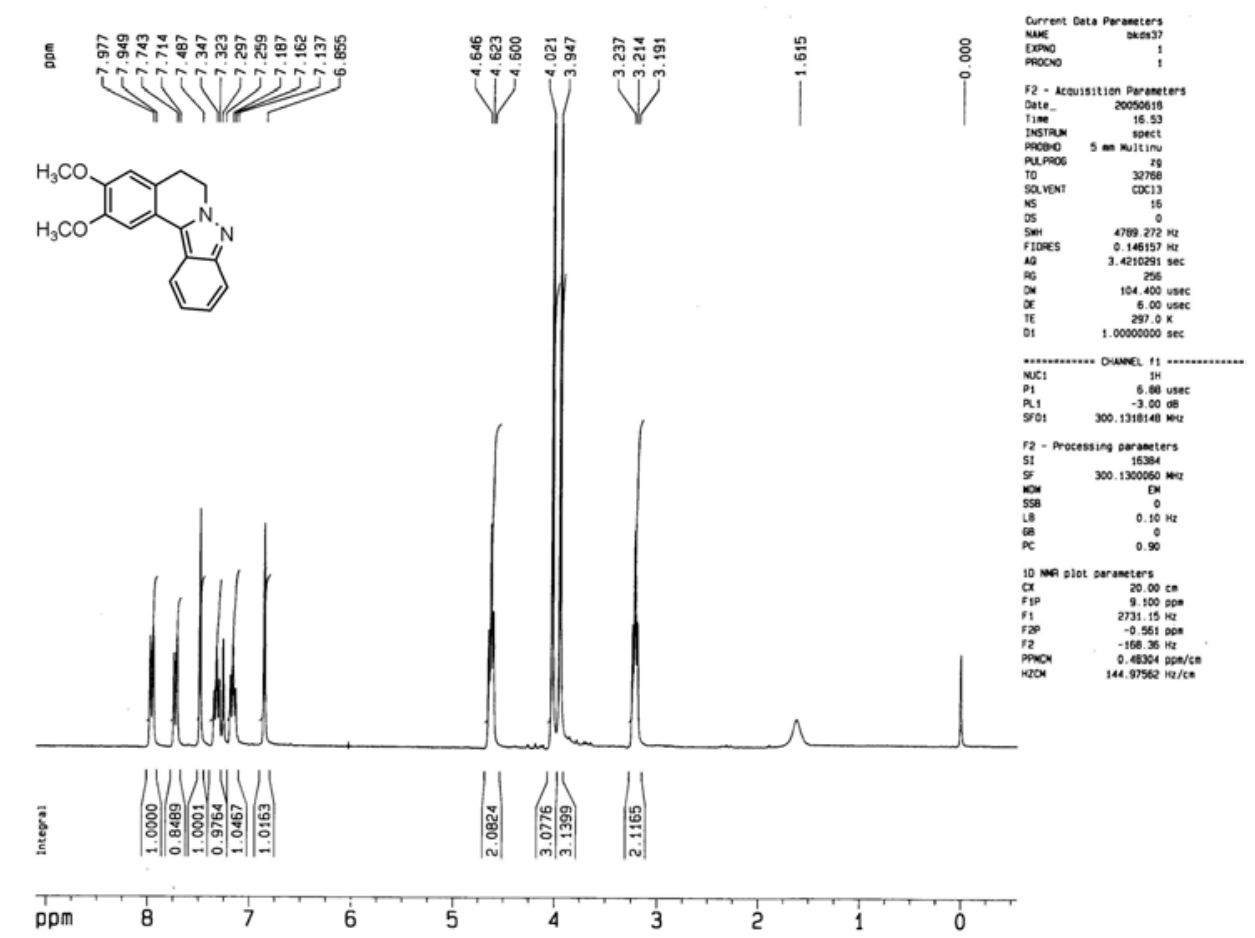

Fig 7: ${ }^{1} \mathrm{H}$ NMR of 2,3-dimethoxy-5,6-dihydroindazolo[3,2-a]isoquinoline [3a].

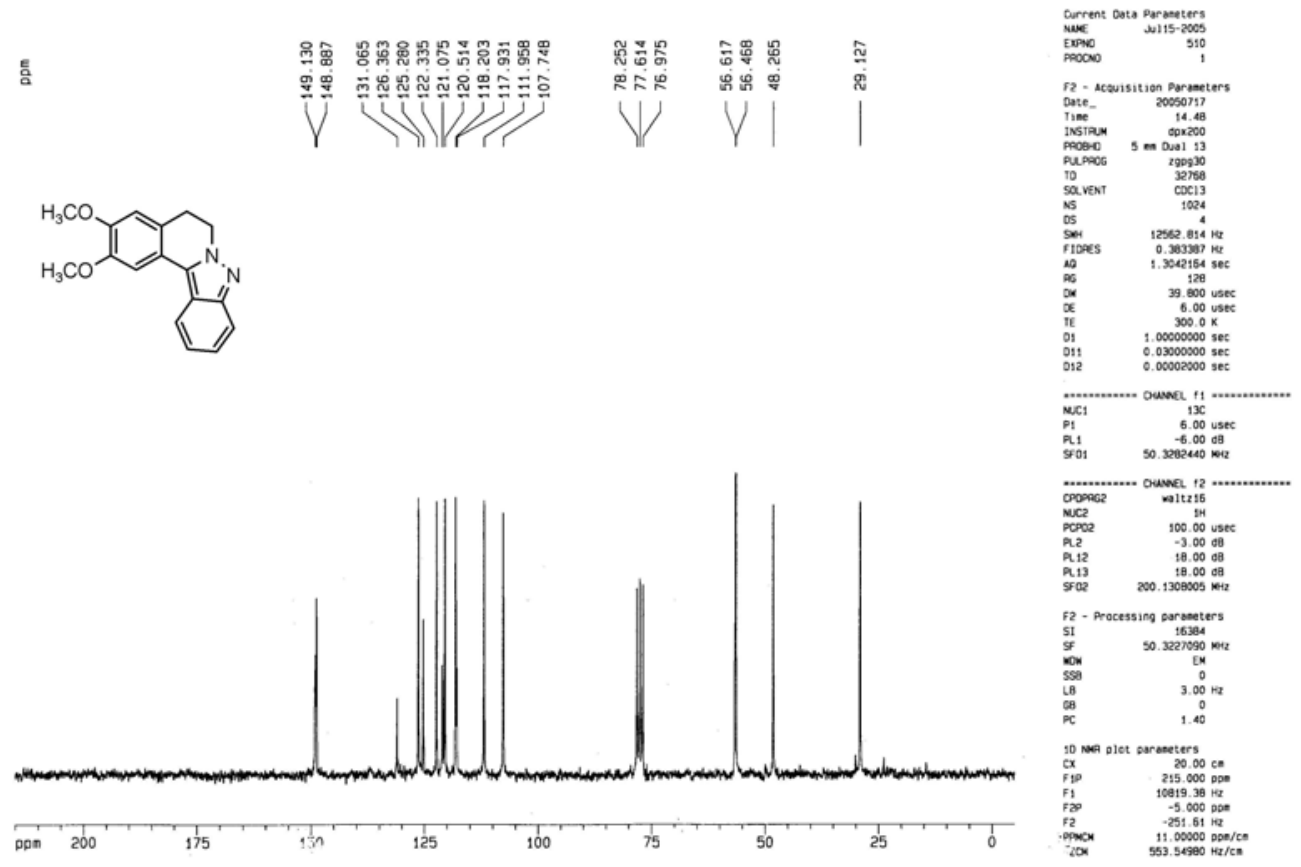

Fig 8: ${ }^{13} \mathrm{C}$ NMR of 2,3-dimethoxy-5,6-dihydroindazolo[3,2-a]isoquinoline [3a]. 


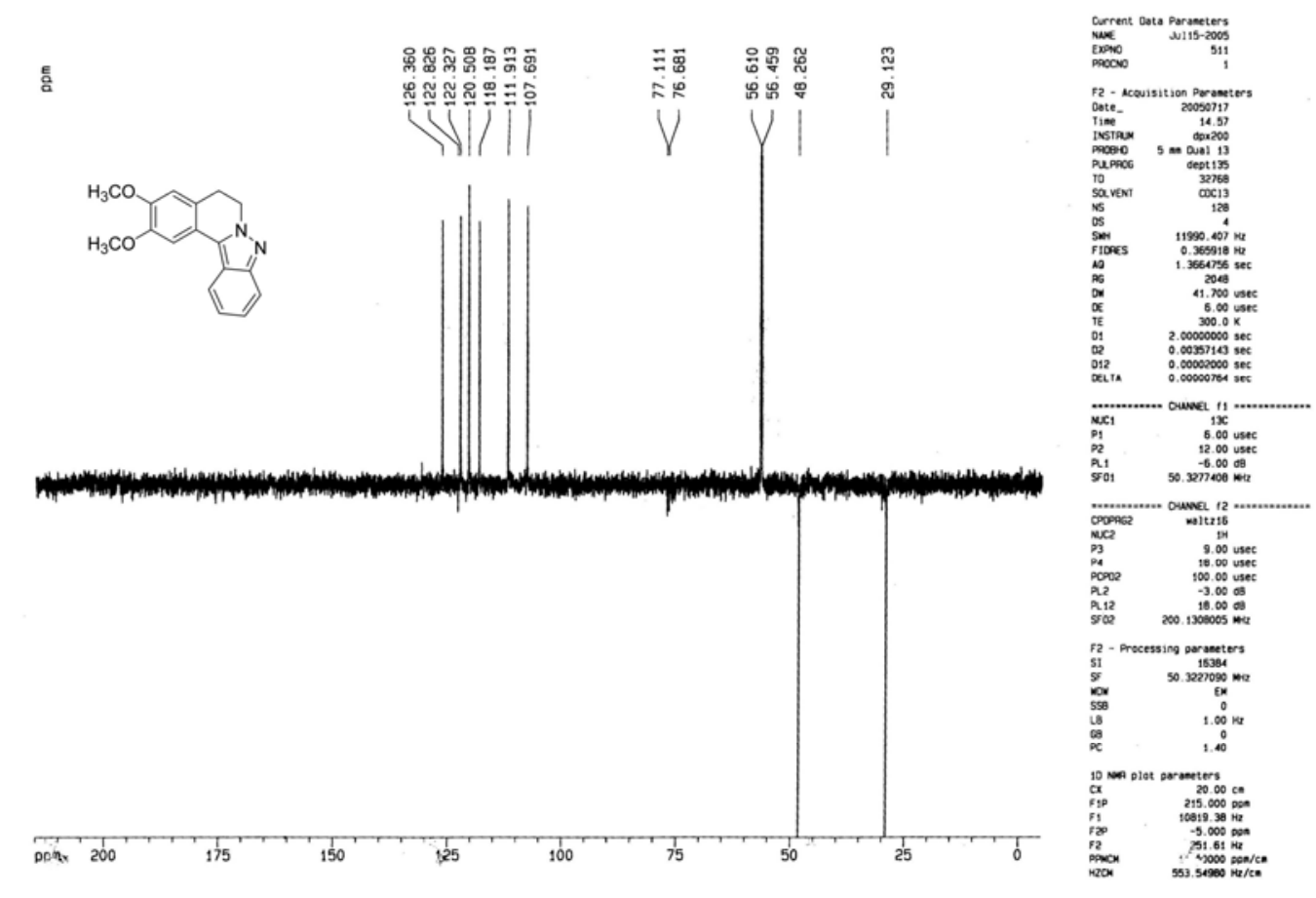

Fig 9: Dept-135 NMR of 2,3-dimethoxy-5,6-dihydroindazolo[3,2-a]isoquinoline [3a]

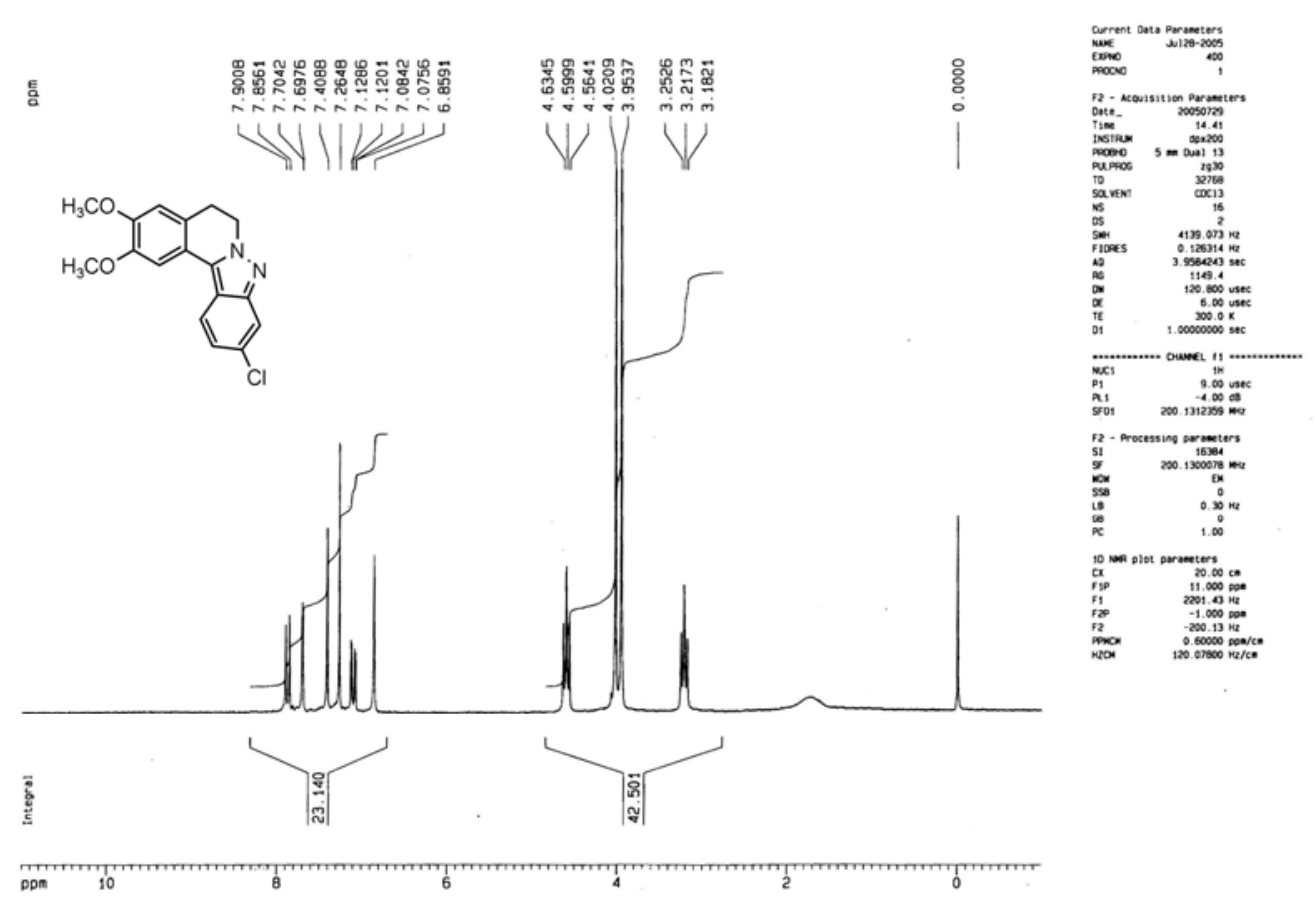

Fig 10: ${ }^{1} \mathrm{H}$ NMR of 10-Chloro-2,3-dimethoxy-5,6-dihydroindazolo[3,2-a]isoquinoline [3b] 

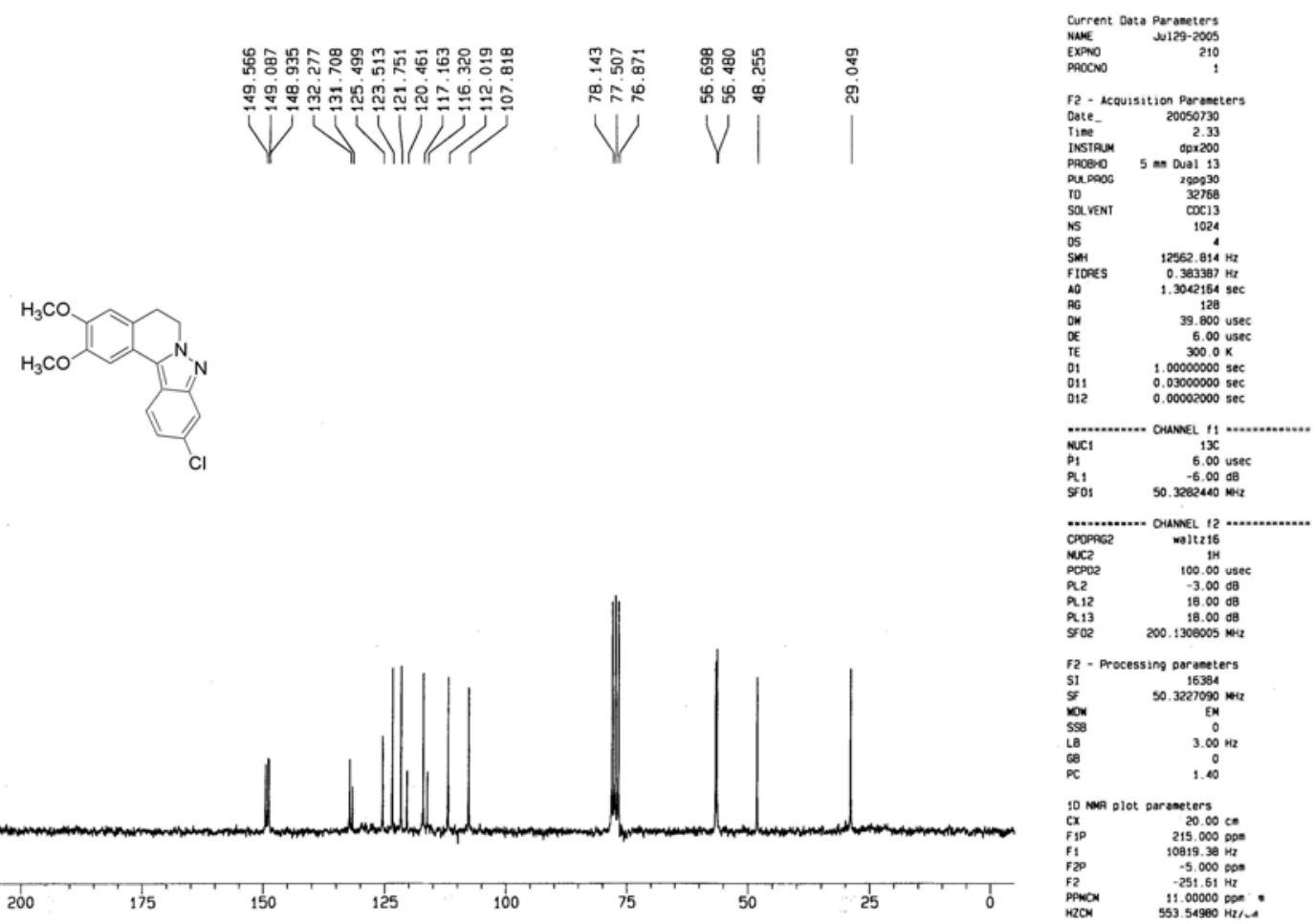

Fig 11: ${ }^{13} \mathrm{C}$ NMR of 10-Chloro-2,3-dimethoxy-5,6-dihydroindazolo[3,2-a]isoquinoline [3b]
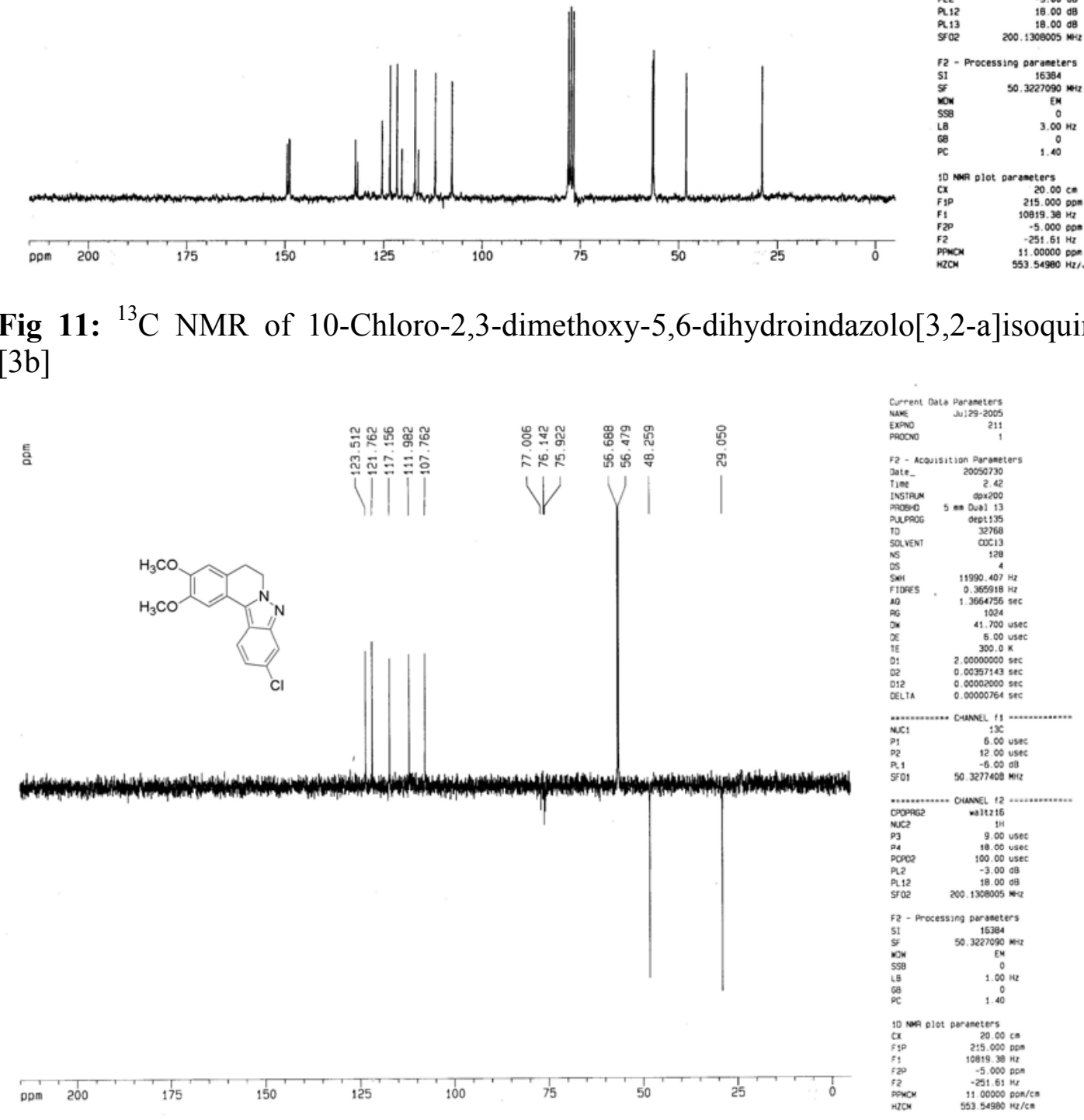

Fig 12: Dept-135 NMR of 10-Chloro-2,3-dimethoxy-5,6-dihydroindazolo[3,2a]isoquinoline [3b] 


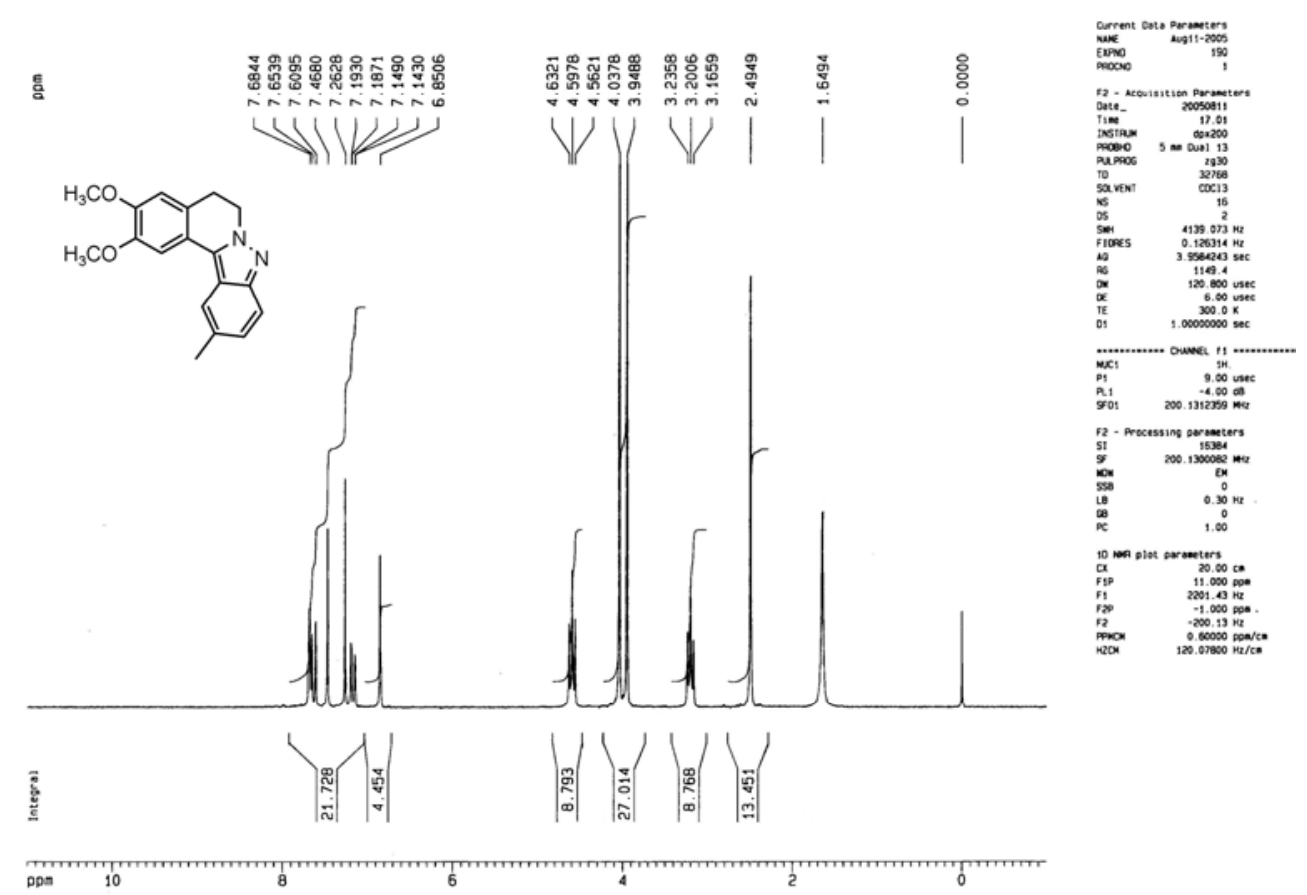

Fig 13: ${ }^{1} \mathrm{H}$ NMR of 2,3-Dimethoxy-11-methyl-5,6-dihydroindazolo[3,2a]isoquinoline [3c]

高

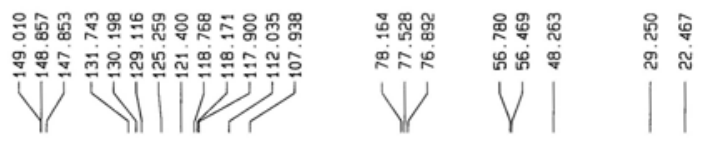
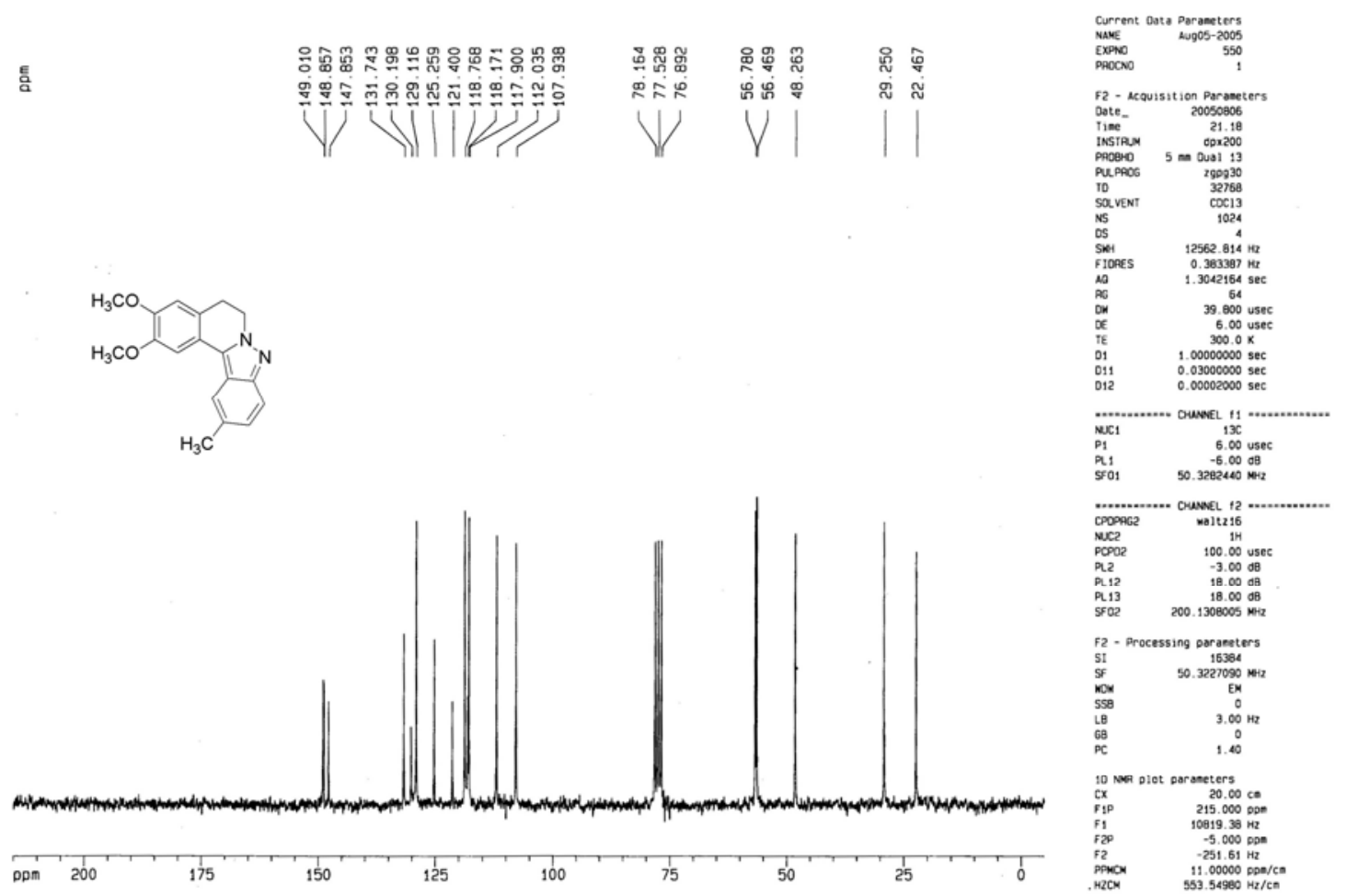

Fig 14: ${ }^{13} \mathrm{C}$ NMR of 2,3-Dimethoxy-11-methyl-5,6-dihydroindazolo[3,2a]isoquinoline [3c] 


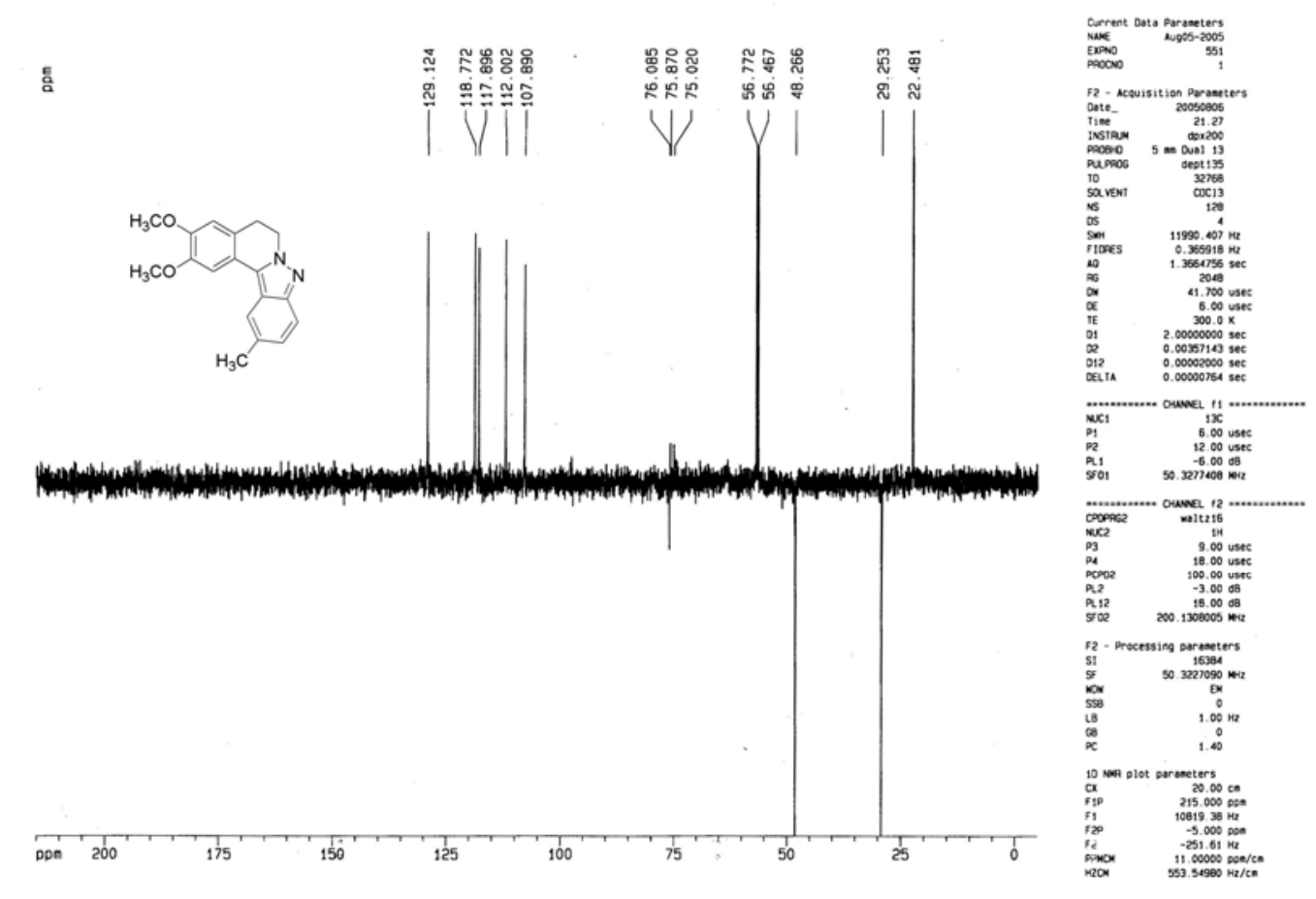

Fig 15: Dept-135 NMR of 2,3-Dimethoxy-11-methyl-5,6dihydroindazolo[3,2a]isoquinoline [3c]

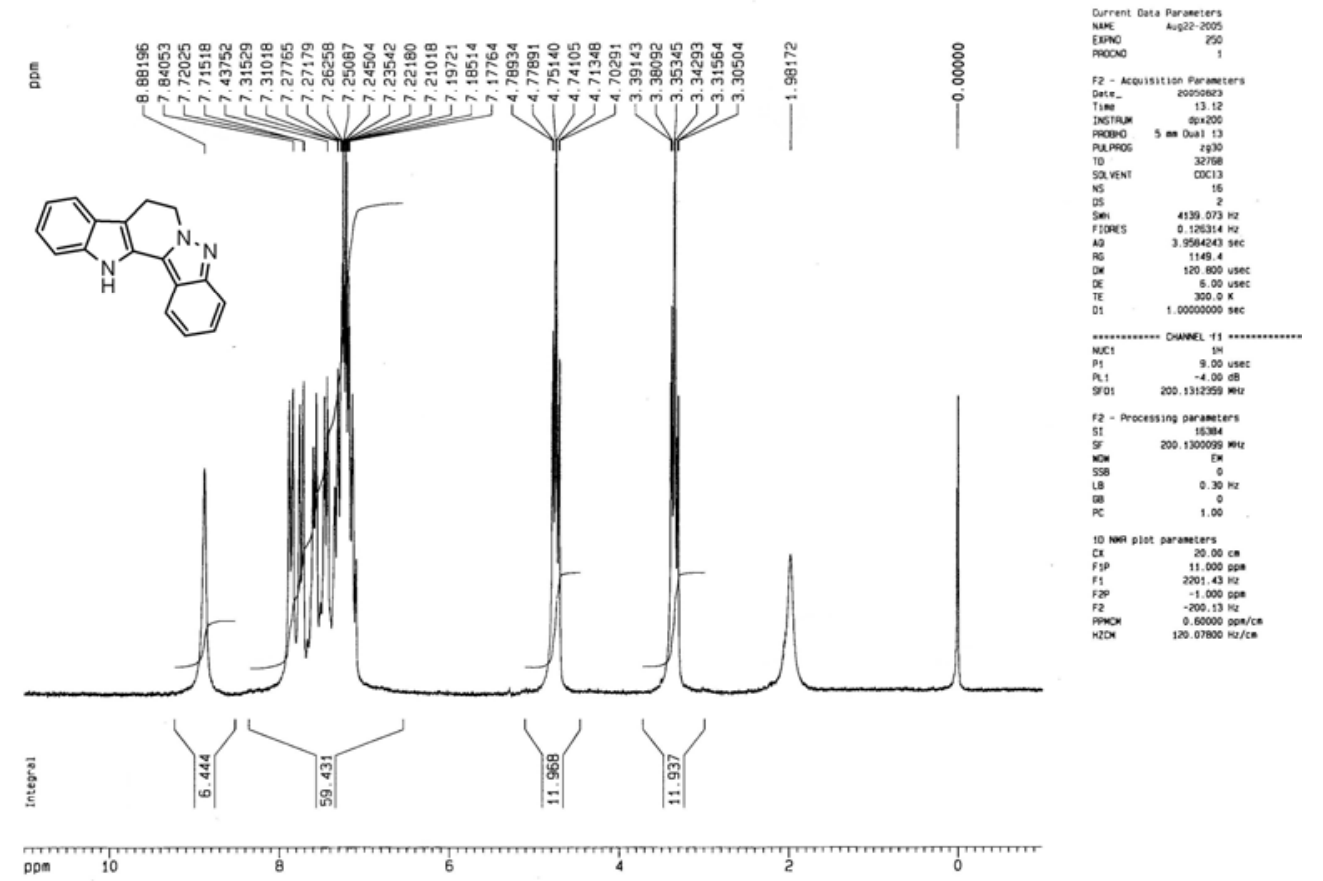

Fig 16: ${ }^{1} \mathrm{H}$ NMR of 5,12-Dihydro-6H-6a,7,12-triazaindeno[1,2a]fluorene [14a] 

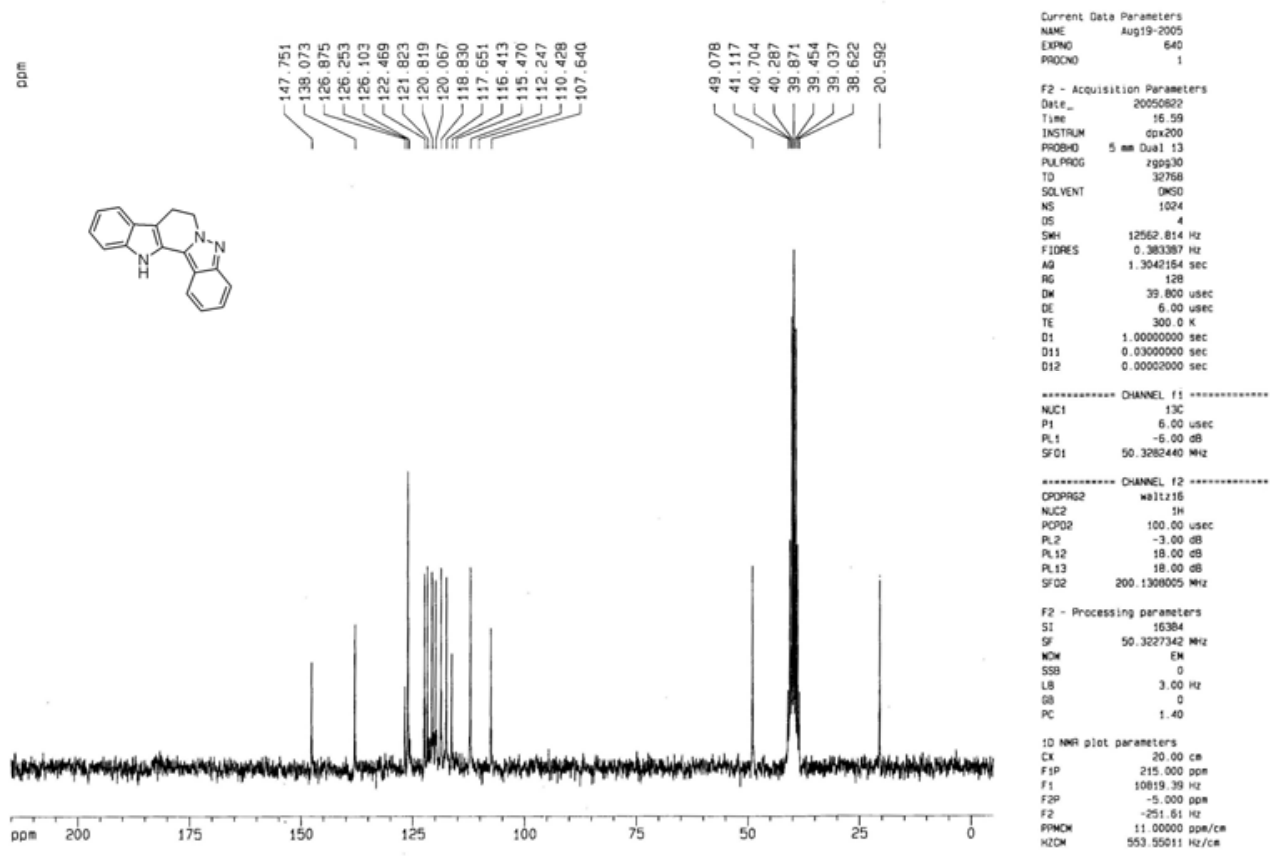

Fig 17: ${ }^{13} \mathrm{C}$ NMR of 5,12-Dihydro-6H-6a,7,12-triazaindeno[1,2a]fluorene [14a]

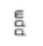
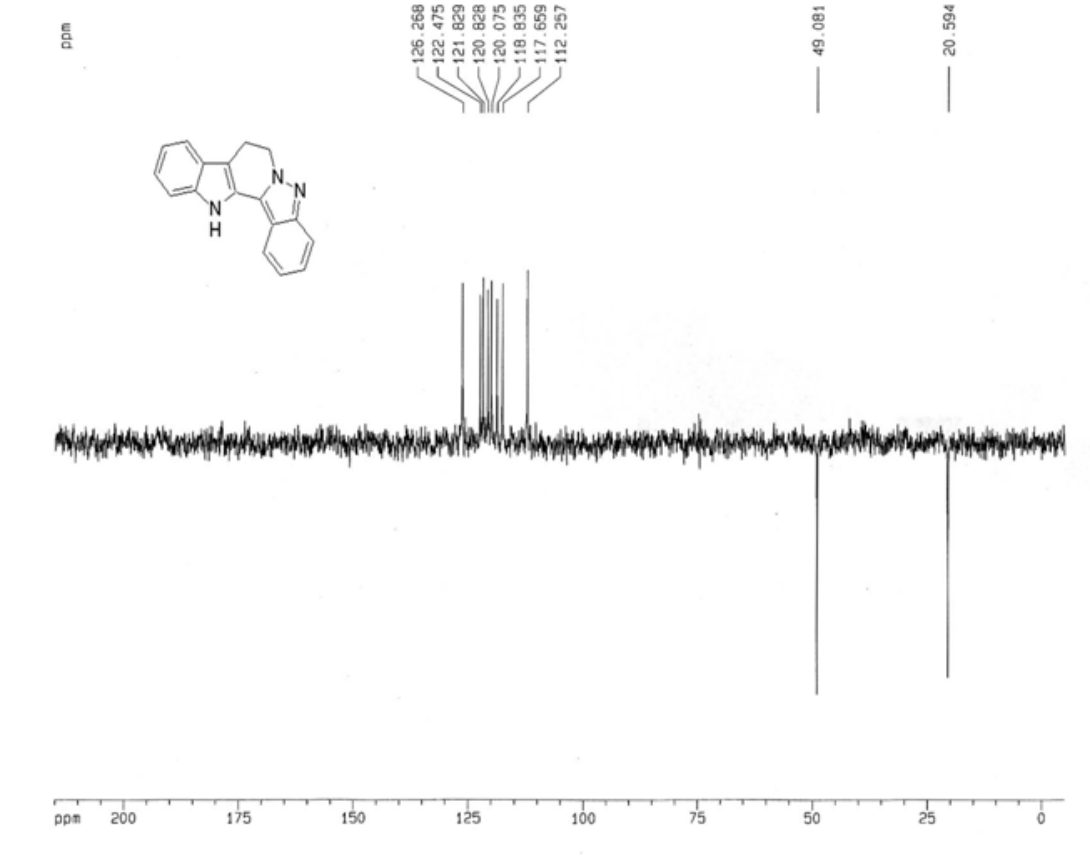

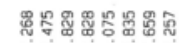

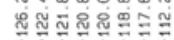

(1)

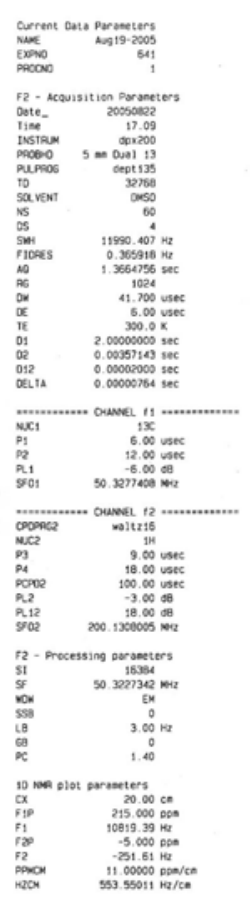

Fig 18: Dept-135 NMR of 5,12-Dihydro-6H-6a,7,12-triazaindeno[1,2a]fluorene [14a] 


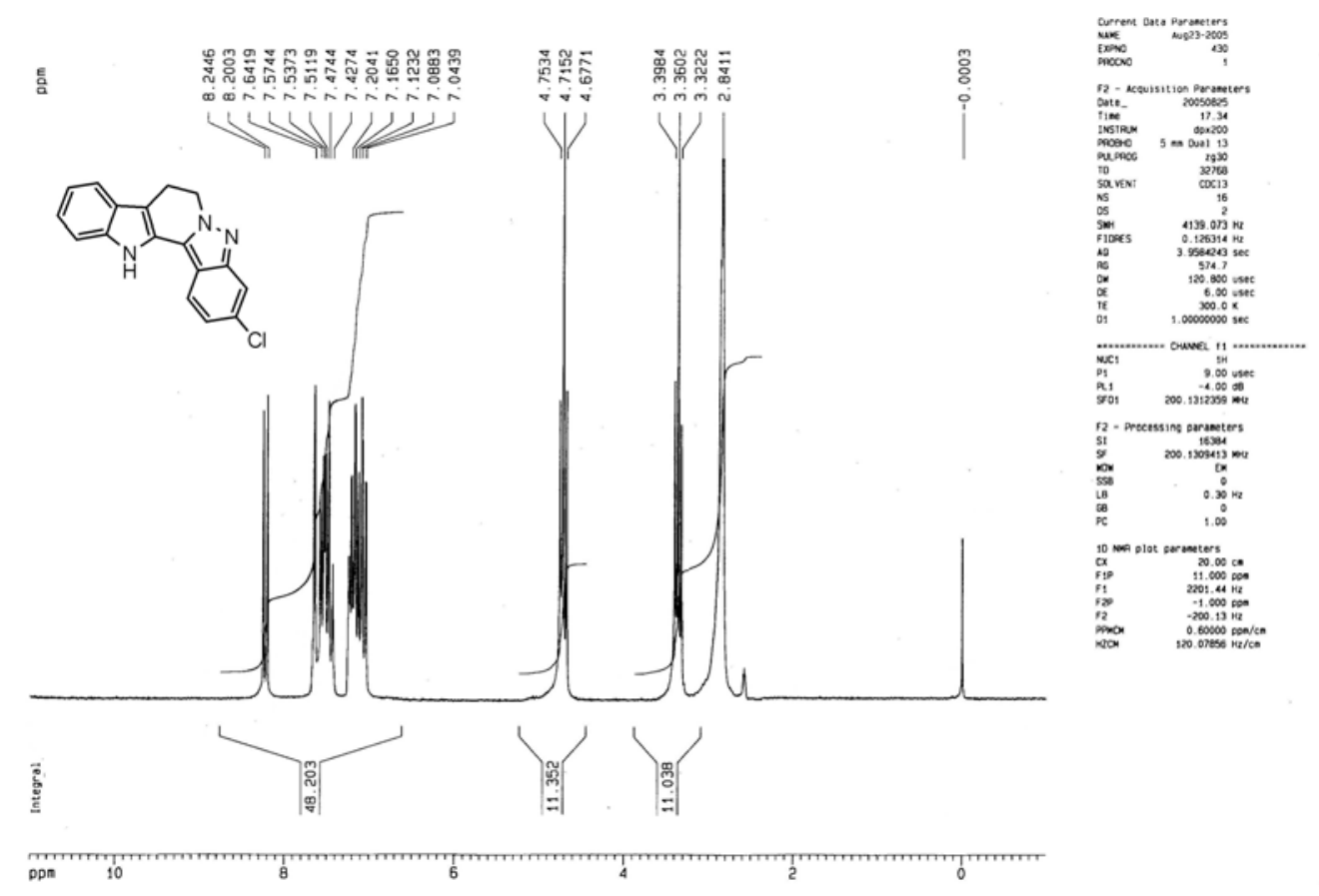

Fig 19: ${ }^{1} \mathrm{H}$ NMR of 9-Chloro-5,12-dihydro-6H-6a,7,12-triazaindeno[1,2-a]fluorene [14b]

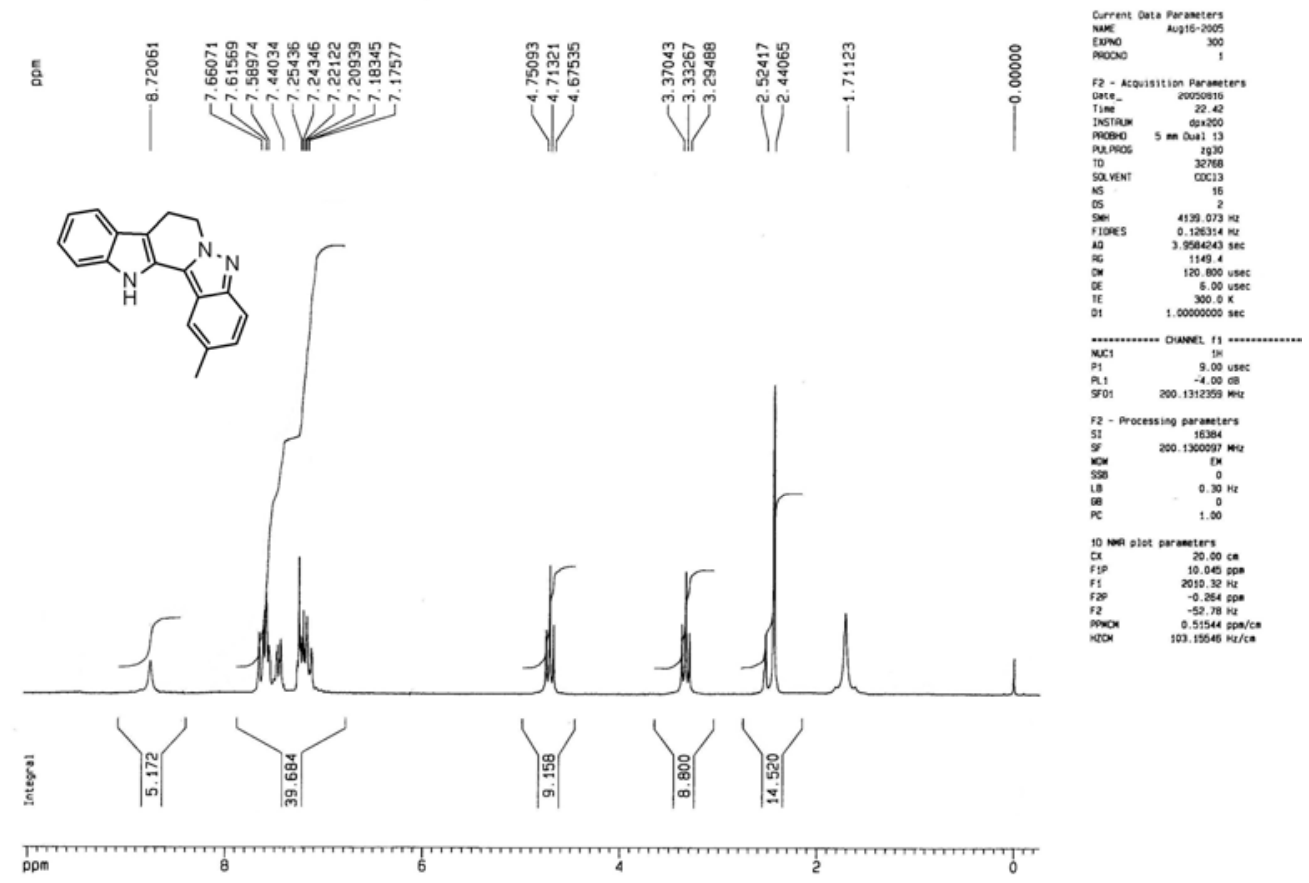

Fig 20: ${ }^{1} \mathrm{H}$ NMR of 10-methyl-5,12-dihydro-6H-6a,7,12-triaza-indeno[1,2-a]fluorene [14c] 


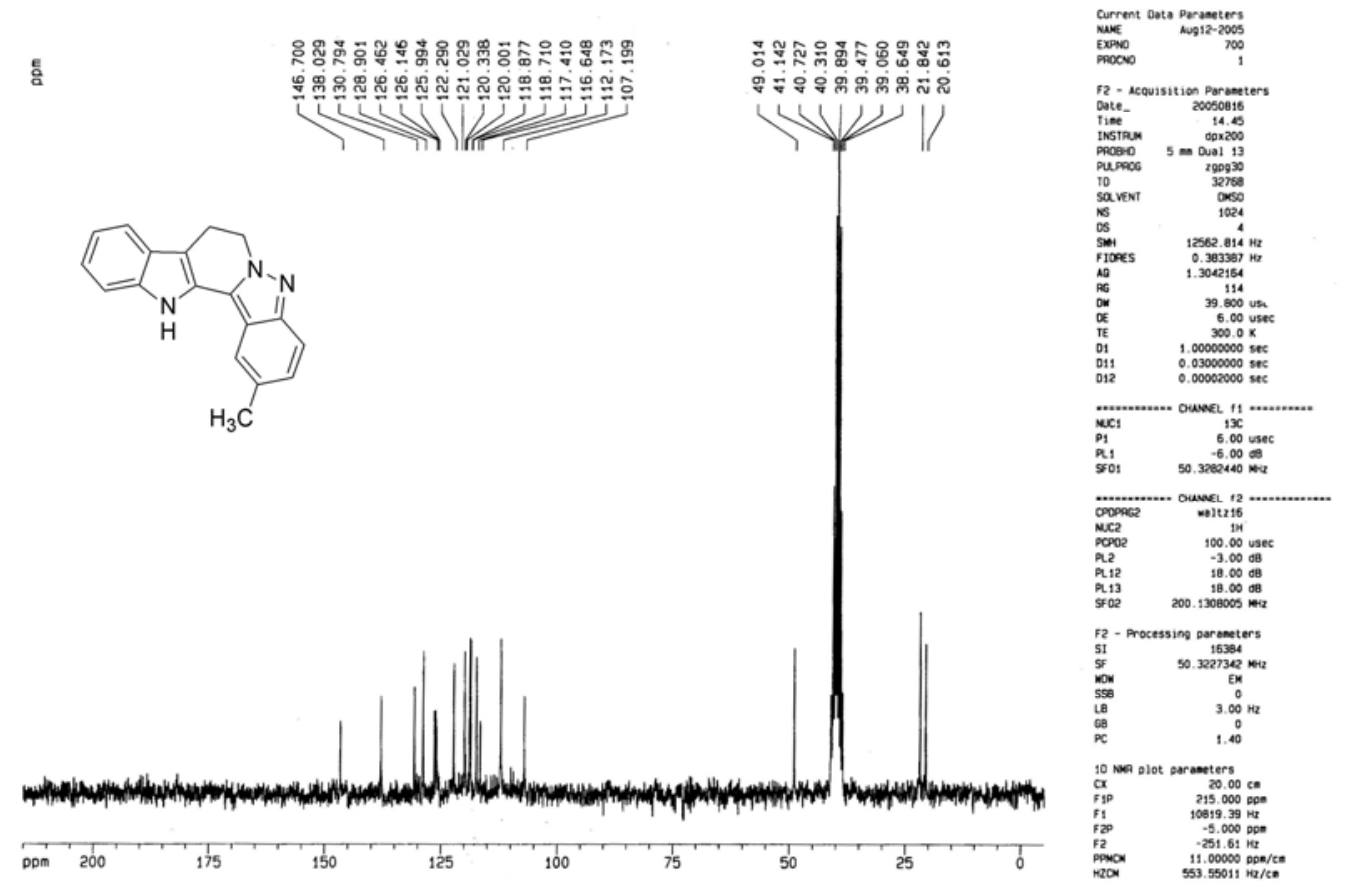

Fig 21: ${ }^{13} \mathrm{C}$ NMR of 10-methyl-5,12-dihydro-6H-6a,7,12-triaza-indeno[1,2-a]fluorene [14c]
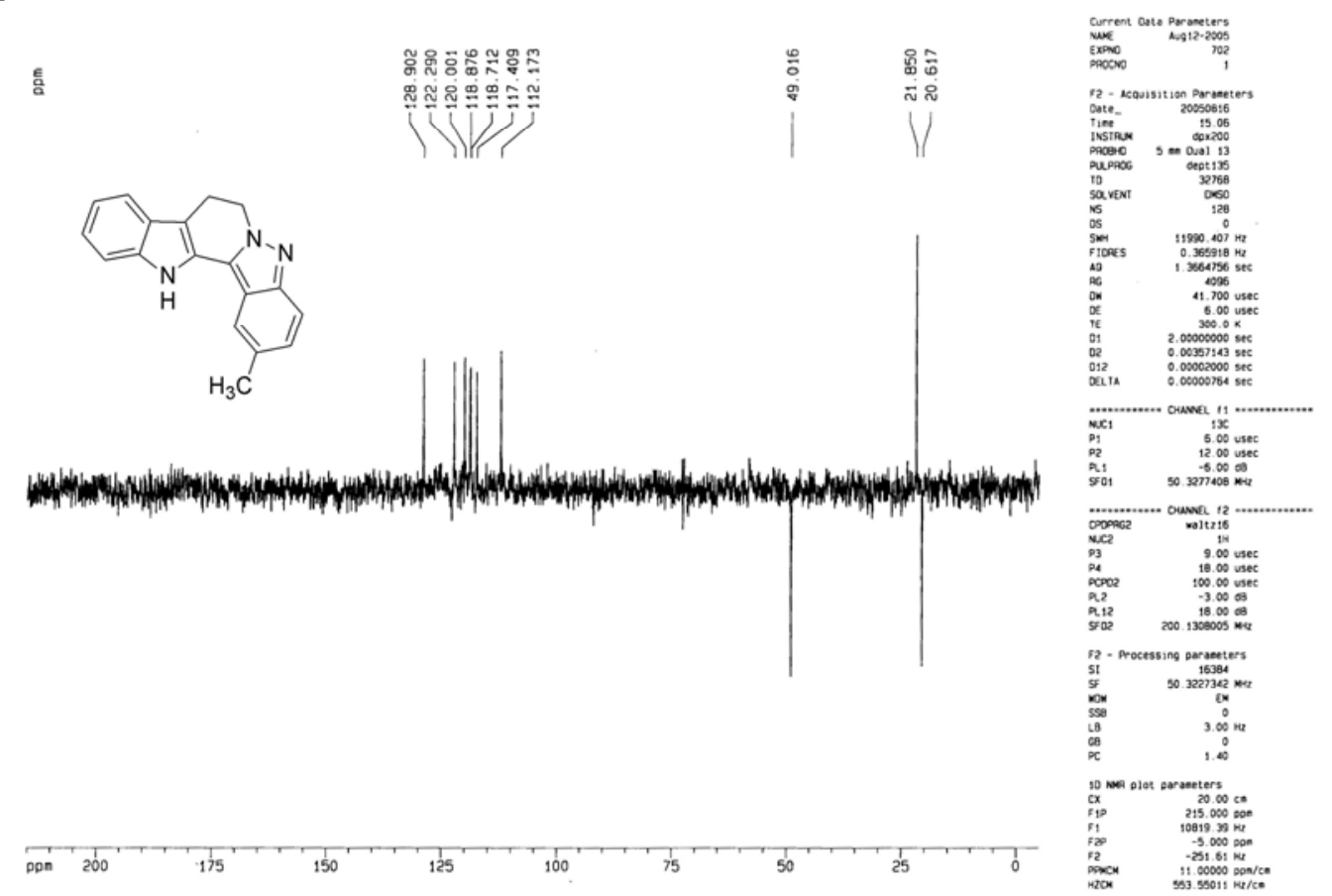

Fig 22: Dept-135 NMR of 10-methyl-5,12-dihydro-6H-6a,7,12-triaza-indeno[1,2a]fluorene [14c] 


\section{X-ray crystallographic data for compounds $3 a$ and $14 a$.}

Crystal data for 3a: $\mathrm{C}_{17} \mathrm{H}_{16} \mathrm{~N}_{2} \mathrm{O}_{2}, M=280.32$, monoclinic, $P 2_{1} / \mathrm{c}, a=11.438(1), b=$ 7.421(1), $c=16.332(1) \AA, \beta=98.35(1)^{\circ}, V=1371.6(2) \AA^{3}, \mathrm{~T}=293(2) \mathrm{K}, Z=4, D_{\mathrm{c}}=$ $1.357 \mathrm{gcm}^{-3}, \mu=0.09 \mathrm{~mm}^{-1}, \lambda\left(\mathrm{Mo} \mathrm{K}_{\alpha}\right)=0.71073 \AA$, transparent block, crystal size 0.325 x $0.250 \times 0.250 \mathrm{~mm}, R 1=0.0478$ for $1681 F o>4 \sigma(F o)$ and 0.0740 for all 2411 data, 193 parameters. CCDC No. 299440;

Crystal data for 14a: $\mathrm{C}_{17} \mathrm{H}_{13} \mathrm{~N}_{3}, M=259.30$, monoclinic, $\mathrm{P} 2_{1} / \mathrm{n}, a=12.167(1), b=$ 17.406(3), $c=12.419(1) \AA, \beta=93.74(1)^{\circ}, V=2624.5(5) \AA^{3}, \mathrm{~T}=293(2) \mathrm{K}, Z=8, D_{\mathrm{c}}=$ $1.313 \mathrm{gcm}^{-3}, \mu=0.08 \mathrm{~mm}^{-1}, \lambda\left(\mathrm{Mo} \mathrm{K}_{\alpha}\right)=0.71073 \AA$, yellow block, crystal size $0.175 \mathrm{x}$ $0.375 \times 0.225 \mathrm{~mm}, R 1=0.0761$ for $1929 F o>4 \sigma(F o)$ and 0.2041 for all 4604 data, 361 parameters. CCDC No. 299441.

(a)
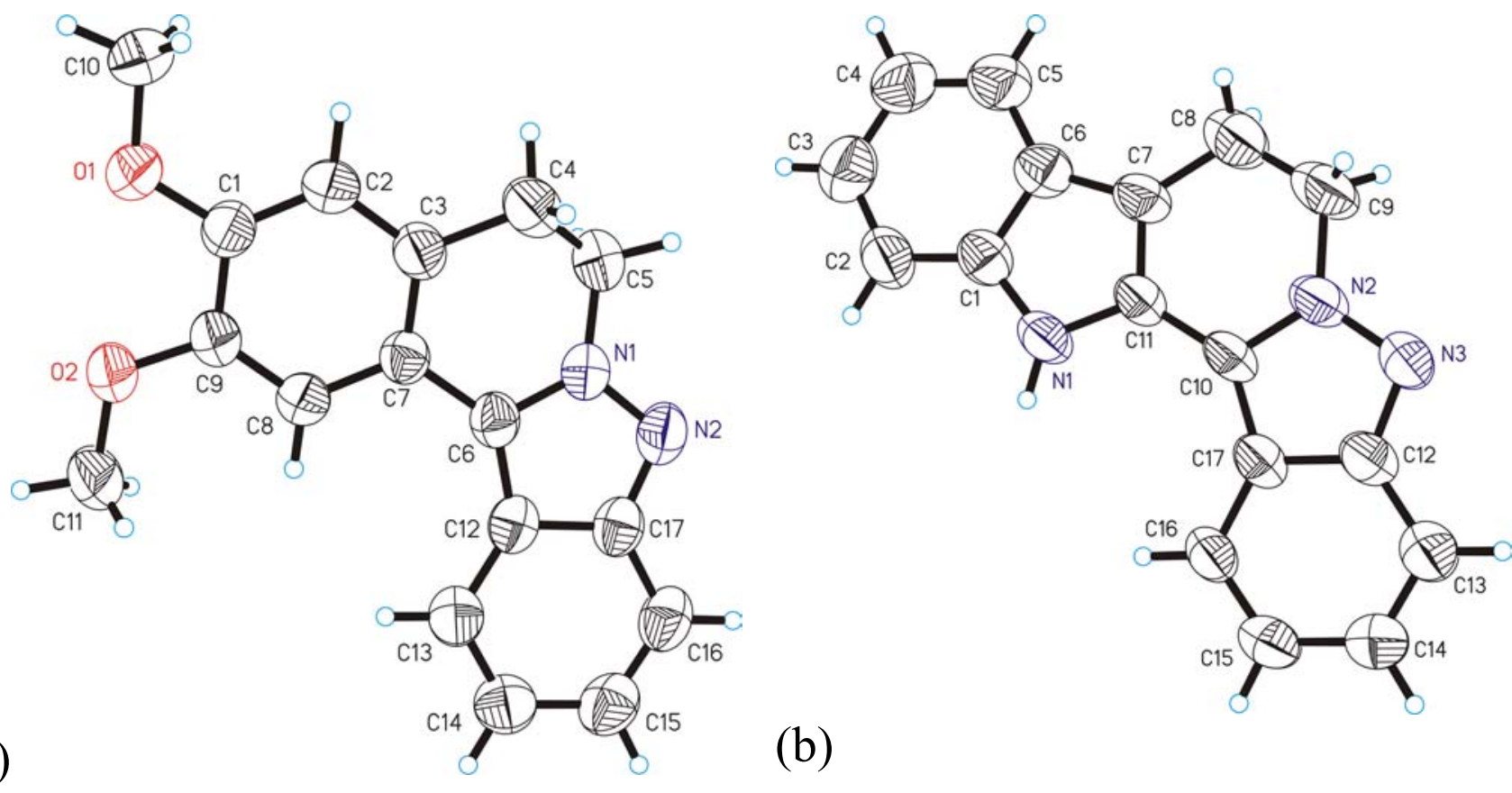

(b)

Fig 2. The ORTEP diagrams (thermal ellipsoids at 50\% probability level) showing the molecular structures and their conformation of (a) ** and (b) **. The atom numbering was given arbitrarily. 


\section{References}

1 Wang, Y. -C.; Georghiou, P. E. Synthesis 2002, 2187.

${ }^{2}$ Yuan, G.;Tang, F. Arkivoc 2003, ii, 32.

${ }^{3}$ Ott, H.; Leslie, G. H.; Trapold, J. H. J. Med. Chem. 1968, 11, 777.

${ }^{4}$ Cortes, E. C.; Romere, E. C.; Ramirez, G. F. J. Heterocycl. Chem. 1994, 31, 1425.

5 Nyerges, M.; Viranyi, A.; Zhang, W.; Groundwater, P. W.; Blasko B.; Toke, L. Tetrahedron 2004, 60, 9937.

${ }^{6}$ Bartra, M.; Romea, P.; Urpi, F.; Vilarrasa, J. Tetrahedron 1990, 46, 587. 